\title{
Tensor Method for Treating the Propagation of Scalar and Electromag- netic Gaussian Schell-Model Beams: A Review
}

\author{
Yangjian $\mathrm{Cai}^{*}$ and Fei Wang \\ School of Physical Science and Technology, Soochow University, Suzhou 215006, China
}

\begin{abstract}
In this paper, we give a review of the tensor method for treating the propagation of scalar and electromagnetic Gaussian Schell-model (GSM) beams. Partially coherent complex curvature tensor is introduced to describe a scalar astigmatic partially coherent GSM beam with twisted phase (i.e., twisted anisotropic GSM beam). A tensor ABCD law for treating the propagation of scalar twisted anisotropic GSM beam through a paraxial astigmatic optical system is derived. The tensor method is then applied to treat the propagation of a scalar twisted anisotripic GSM beam through an apertured optical system, fractional Fourier transform optical system, dispersive and absorbing media, turbulent media. Furthermore, we applied the tensor method to treat the propagation of an electromagnetic GSM beam. Propagation formulae for an electromagnetic GSM beam passing through a paraxial astigmatic optical system in free space and in turbulent atmosphere are derived. The propagation properties of an electromagnetic GSM beam in a Gaussian cavity and in turbulent atmosphere are also reviewed.
\end{abstract}

Keywords: Partially coherent beam, stochastic electromagnetic beam, tensor method, propagation.

\section{INTRODUCTION}

Partially coherent beams have important applications in free-space optical communications, inertial confinement fusion, optical imaging, optical projection, laser scanning, photography, nonlinear optics and optical trapping [1-13]. In the past decades, especially since Wolf first found that a partially coherent beam undergoes a spectral shift during its propagation in free space [14-16], partially coherent beams have been extensively studied both in experiment and theory.

Conventionally scalar partially coherent beams are characterized by a second-order correlation function of the electric field which depends on two spatial arguments and the oscillation frequency and being called the cross-spectral density (CSD) [1]. Gaussian Schell-model (GSM) beam is a typical partially coherent beam, whose spectral density and spectral degree of coherence have Gaussian shapes [17-22]. By scattering a coherent laser beam from a rotating grounded glass, then transforming the spectral density distribution of the scattered light into Gaussian profile with a Gaussian amplitude filter, a GSM beam can be generated [17, 22]. GSM beams can also be generated with specially synthesized rough surfaces, spatial light modulators and synthetic acousto-optic holograms (c.f. [23]).

A more general partially coherent beam can possess a twist phase, which differs in many respects from the customary quadratic phase factor. Simon and Mukunda first introduced the partially coherent twisted Gaussian Schellmodel (GSM) beam opening up "a new dimension" in the area of partially coherent fields [24-26]. Unlike the usual phase curvature, the twist phase is bounded in strength due to the fact that the cross-spectral density function must be

*Address correspondence to this author at the School of Physical Science and Technology, Soochow University, Suzhou 215006, China; Fax: +86 512 65111907; Tel: +86 512 65244275; E-mail: yangjian_cai@yahoo.com.cn nonnegative and, moreover, it is absent in a coherent Gaussian beam [24-28]. The twist phase has an intrinsic chiral or handedness property and is responsible for the rotation of the beam spot on propagation [24-30]. An essential aspect of the twist phase is that it is intrinsically twodimensional, and it cannot be separated into a sum of onedimensional contributions [24-30]. Experimental observation of the twisted GSM beam has been reported in Ref. [30]. Later the studies relating to coherent-mode decomposition [31], the analysis of the transfer of radiance [32], radiation efficiency [33], the orbital angular momentum [34] of the twisted GSM beams have been carried out.

The conventional method for treating the propagation of GSM and twisted GSM beams is Wigner-distribution function [24-28, 35, 36]. Lin and Cai have introduced a convenient alternative tensor method for treating propagation of GSM and twisted GSM beams [37]. With the help of the tensor method, paraxial propagation of the GSM and twisted GSM beams through free space, paraxial optical systems, dispersive and absorbing media, turbulent atmosphere, have been then studied in details [38-46]. The influence of the twist phase on the second-harmonic generation by a twisted GSM beam has been investigated [11]. Ghost imaging with GSM and twisted GSM beams was examined recently [6, 47].

Polarization is another important property of a laser beam. In the past decades the two important properties of light waves: coherence and polarization were studied separately (cf. [1], [48]). Only a few years ago it was found that the degree of polarization of a stochastic electromagnetic beam (i.e., partially coherent and partially polarized beam) may change on propagation, and such changes depend on the coherence properties of the source of the beam [49-51]. The electromagnetic GSM beams (also called partially coherent and partially polarized GSM beams or vectorial GSM beams) were introduced theoretically as the natural extension of the scalar GSM beams [52-55]. In the past several years, 
electromagnetic GSM beam has been investigated widely due to its importance in theories of coherence and polarization and in some applications, e.g. free-space optical communications [56-71]. Several methods have later been proposed for their synthesis $[56,57]$. The realizability conditions, i.e. conditions that the parameters of the source should satisfy to produce a physically realizable field, have also been established for electromagnetic GSM beam [58, 59]. Propagation of electromagnetic GSM beams through free space, turbulent atmosphere, human tissue and gradientindex fiber have been extensively studied [60-71]. Cai and co-workers have applied the tensor method to obtain the analytical propagation formulae for electromagnetic GSM beams passing through paraxial ABCD optical system in free space and in turbulent atmosphere [72-74], and investigated the evolution properties of electromagnetic GSM beam in dispersive media and in resonators [75-78]. Furthermore, they applied electromagnetic GSM beam for Lidar systems in turbulent atmosphere with the derived formulae [79]. With the help of tensor method, twist phase-induced polarization changes in electromagnetic Gaussian Schell-model beams were studied recently [80].

In this paper, we review our work done about the propagation of scalar and electromagnetic GSM beams by introducing the convenient tensor method.

\section{PARTIALLY COHERENT COMPLEX CURVATURE TENSOR AND TENSOR ABCD LAW}

The cross-spectral density of a scalar twisted anisotropic GSM beam is expressed as [24-31]

$$
\begin{aligned}
& \mathrm{W}\left(\mathbf{r}_{1}, \mathbf{r}_{2}\right)= \\
& \begin{aligned}
G_{0} \exp \{- & \frac{1}{4}\left[\mathbf{r}_{1}^{T}\left(\hat{o}_{I}^{2}\right)^{-1} \mathbf{r}_{1}+\mathbf{r}_{2}^{T}\left(\hat{o}_{I}^{2}\right)^{-1} \mathbf{r}_{2}\right] \\
& -\frac{1}{2}\left(\mathbf{r}_{1}-\mathbf{r}_{2}\right)^{T}\left(o_{g}^{2}\right)^{-1}\left(\mathbf{r}_{1}-\mathbf{r}_{2}\right) \\
& \left.-\frac{i k}{2}\left(\mathbf{r}_{1}-\mathbf{r}_{2}\right)^{T}\left(\mathbf{R}^{-1}+\mu \mathbf{J}\right)\left(\mathbf{r}_{1}+\mathbf{r}_{2}\right)\right\},
\end{aligned}
\end{aligned}
$$

where $G_{0}$ is a constant, $\mathbf{r}_{1}$ and $\mathbf{r}_{2}$ are position vectors of two arbitrary points in the transverse plane. $k=2 \pi / \lambda$ is the wave number with $\lambda$ being the wavelength of light field. $o_{I}^{2}$ and $\delta_{g}^{2}$ are transverse spot width matrix and transverse coherence width matrix, respectively, and they have dimension of length square. $\mathbf{R}^{-1}$ is wave front curvature matrix with dimension of inverse length. $\delta_{I}^{2}, \delta_{g}^{2}$ and $\mathbf{R}^{-1}$ are all $2 \times 2$ matrices with transpose symmetry. $\mathbf{J}$ is a transpose anti-symmetric matrix given by:

$\mathbf{J}=\left(\begin{array}{cc}0 & 1 \\ -1 & 0\end{array}\right)$

$\mu$ is a scalar real-valued twist factor with inverse length dimension.

Eq. (1) can be expressed in following alternative tensor form [37]
$\mathrm{W}(\tilde{\mathbf{r}})=G_{0} \exp \left(-\frac{i k}{2} \tilde{\mathbf{r}}^{T} \mathbf{M}_{1}^{-1} \tilde{\mathbf{r}}\right)$,

$\mathbf{M}_{1}^{-1}$ is a $4 \times 4$ matrix, called partially coherent complex curvature tensor, takes the form

$\mathbf{M}_{1}^{-1}=\left(\begin{array}{cc}\mathbf{R}^{-1}-\frac{i}{2 k}\left(\hat{o}_{I}^{2}\right)^{-1}-\frac{i}{k}\left(\hat{o}_{g}^{2}\right)^{-1} & \frac{i}{k}\left(\hat{o}_{g}^{2}\right)^{-1}+\mu \mathbf{J} \\ \frac{i}{k}\left(\hat{o}_{g}^{2}\right)^{-1}+\mu \mathbf{J}^{T} & -\mathbf{R}^{-1}-\frac{i}{2 k}\left(\dot{o}_{I}^{2}\right)^{-1}-\frac{i}{k}\left(\delta_{g}^{2}\right)^{-1}\end{array}\right)$

If we set $\mu=0$ in Eq. (4), Eq. (3) can be used to describe an anisotropic GSM beam. If $\mathbf{M}_{1}^{-1}$ takes the following form

$$
\mathbf{M}_{1}^{-1}=\left(\begin{array}{cc}
\left(-\frac{i}{2 k \sigma_{I}^{2}}-\frac{i}{k \sigma_{g}^{2}}\right) \mathbf{I} & \frac{i}{k \sigma_{g}^{2}} \mathbf{I}+\mu \mathbf{J} \\
\frac{i}{k \sigma_{g}^{2}} \mathbf{I}+\mu \mathbf{J}^{T} & \left(-\frac{i}{2 k \sigma_{I}^{2}}-\frac{i}{k \sigma_{g}^{2}}\right) \mathbf{I}
\end{array}\right)
$$

where $I$ is a $2 \times 2$ unit matrix, $\sigma_{I}$ and $\sigma_{g}$ denote the transverse beam width and spectral coherence width, respectively, Eq. (3) can be used to describe a twisted isotropic GSM beam. If we set $\mu=0$ in Eq. (5), Eq. (3) can be used to describe a isotropic GSM beam.

After propagation through a general astigmatic (i.e., nonsymmetrical) ABCD optical system, the cross-spectral density of the GSM beam in the output plane is expressed as follows [37]

$\mathrm{W}(\tilde{\mathbf{n}})=\frac{G_{0}}{\left[\operatorname{det}\left(\tilde{\mathbf{A}}+\tilde{\mathbf{B}} \mathbf{M}_{1}^{-1}\right)\right]^{1 / 2}} \exp \left(-\frac{i k}{2} \tilde{\mathbf{n}}^{T} \mathbf{M}_{2}^{-1} \tilde{\mathbf{n}}\right)$,

where det stands for the determinant of a matrix, $\tilde{\mathbf{n}}^{T}=\left(\begin{array}{cc}\tilde{\mathrm{n}}_{1}^{T} & \tilde{\mathrm{n}}_{2}^{T}\end{array}\right) \infty$ with $\tilde{\mathrm{n}}_{1}$ and $\tilde{\mathrm{n}}_{2}$ being two arbitrary position vectors in the output plane, $\mathbf{M}_{2}^{-1}$ is the partially coherent complex curvature tensor in the output plane, and is related with $\mathbf{M}_{1}^{-1}$ through the following tensor ABCD law [37]

$$
\mathbf{M}_{2}^{-1}=\left(\tilde{\mathbf{C}}+\tilde{\mathbf{D}} \mathbf{M}_{1}^{-1}\right)\left(\tilde{\mathbf{A}}+\tilde{\mathbf{B}} \mathbf{M}_{1}^{-1}\right)^{-1}
$$

where matrices $\tilde{\mathbf{A}}, \tilde{\mathbf{B}}, \tilde{\mathbf{C}}$ and $\tilde{\mathbf{D}}$ have the forms

$$
\begin{aligned}
\tilde{\mathbf{A}} & =\left(\begin{array}{cc}
\mathbf{A} & 0 \mathbf{I} \\
0 \mathbf{I} & \mathbf{A}^{*}
\end{array}\right), \tilde{\mathbf{B}}=\left(\begin{array}{cc}
\mathbf{B} & 0 \mathbf{I} \\
0 \mathbf{I} & -\mathbf{B}^{*}
\end{array}\right), \\
\tilde{\mathbf{C}} & =\left(\begin{array}{cc}
\mathbf{C} & \mathbf{0 I} \\
\mathbf{0 I} & -\mathbf{C}^{*}
\end{array}\right), \tilde{\mathbf{D}}=\left(\begin{array}{cc}
\mathbf{D} & 0 \mathbf{I} \\
0 \mathbf{I} & \mathbf{D}^{*}
\end{array}\right),
\end{aligned}
$$

with $\mathbf{A}, \mathbf{B}, \mathbf{C}$ and $\mathbf{D}$ being the $2 \times 2$ sub-matrices of the astigmatic ABCD optical system. Symbol “*” in Eq. (8) which denotes the complex conjugate is required for a 
general optical system in the presence of loss or gain, although it does not appear in Eq. (13) of Ref. [37].

In practice, most optical systems are slightly misaligned more or less, and it is necessary to take the misalignment of the optical systems into consideration. Following [38], after propagation through a stigmatic (i.e., symmetrical) ABCD optical system, the cross-spectral density of the GSM beam in the output plane is expressed as follows

$$
\begin{aligned}
\mathrm{W}(\tilde{\mathbf{n}})= & \frac{G_{0}}{\left[\operatorname{det}\left(\overline{\mathbf{A}}+\overline{\mathbf{B}} \mathbf{M}_{1}^{-1}\right)\right]^{1 / 2}} \exp \left(\begin{array}{c}
-\frac{i k}{2} \tilde{\mathbf{n}}^{T} \mathbf{M}_{2}^{-1} \tilde{\mathbf{n}}-\frac{i k}{2} \tilde{\mathbf{n}}^{T} \overline{\mathbf{B}}^{-1} \overline{\mathbf{g}}_{h} \\
-\frac{i k}{2} \tilde{\mathbf{n}}^{T} \overline{\mathbf{B}}^{-1 T}\left(\overline{\mathbf{A}}+\overline{\mathbf{B}} \mathbf{M}_{1}^{-1}\right)^{-1} \overline{\mathbf{e}}_{f}
\end{array}\right) \\
& \times \exp \left[\frac{i k}{8} \overline{\mathbf{e}}_{f}^{T} \overline{\mathbf{B}}^{-1 T}\left(\overline{\mathbf{A}}+\overline{\mathbf{B}} \mathbf{M}_{1}^{-1}\right)^{-1} \overline{\mathbf{e}}_{f}\right],
\end{aligned}
$$

where $\mathbf{M}_{2}^{-1}$ is related with $\mathbf{M}_{1}^{-1}$ through the following tensor ABCD law [38]

$\mathbf{M}_{2}^{-1}=\left(\overline{\mathbf{C}}+\overline{\mathbf{D}} \mathbf{M}_{1}^{-1}\right)\left(\overline{\mathbf{A}}+\overline{\mathbf{B}} \mathbf{M}_{1}^{-1}\right)^{-1}$

$\overline{\mathbf{A}}, \overline{\mathbf{B}}, \overline{\mathbf{C}}$ and $\overline{\mathbf{D}}$ are defined as

$$
\begin{aligned}
& \overline{\mathbf{A}}=\left(\begin{array}{cc}
\mathbf{A} & 0 \mathbf{I} \\
0 \mathbf{I} & \mathbf{A}^{*}
\end{array}\right), \overline{\mathbf{B}}=\left(\begin{array}{cc}
\mathbf{B} & 0 \mathbf{I} \\
0 \mathbf{I} & -\mathbf{B}^{*}
\end{array}\right), \\
& \overline{\mathbf{C}}=\left(\begin{array}{cc}
\mathbf{C} & \mathbf{0 I} \\
\mathbf{0 I} & -\mathbf{C}^{*}
\end{array}\right), \overline{\mathbf{D}}=\left(\begin{array}{cc}
\mathbf{D} & 0 \mathbf{I} \\
0 \mathbf{I} & \mathbf{D}^{*}
\end{array}\right),
\end{aligned}
$$

A, B, C and $\mathbf{D}$ take the following form

$$
\begin{array}{ll}
\mathbf{A}=\left(\begin{array}{ll}
a & 0 \\
0 & a
\end{array}\right), & \mathbf{B}=\left(\begin{array}{ll}
b & 0 \\
0 & b
\end{array}\right), \\
\mathbf{C}=\left(\begin{array}{ll}
c & 0 \\
0 & c
\end{array}\right), & \mathbf{D}=\left(\begin{array}{ll}
d & 0 \\
0 & d
\end{array}\right),
\end{array}
$$

where $a, b, c$ and $d$ are the transfer matrix elements of aligned stigmatic optical system. $\quad \overline{\mathbf{e}}_{f}^{T}=\left(\begin{array}{cc}\mathbf{e}_{f}^{T} & \mathbf{e}_{f}^{T}\end{array}\right)$, $\overline{\mathbf{g}}_{h}^{T}=\left(\begin{array}{ll}\mathbf{g}_{h}^{T} & \mathbf{g}_{h}^{T}\end{array}\right), \mathbf{e}_{f}=\left(\begin{array}{ll}e & f\end{array}\right), \mathbf{g}_{h}=\left(\begin{array}{ll}g & h\end{array}\right), e, f, g$ and $h$ take the following form

$$
\begin{aligned}
& e=2\left(\alpha_{T} \varepsilon_{x}+\beta_{T} \varepsilon_{x}^{\prime}\right), \\
& f=2\left(\alpha_{T} \varepsilon_{y}+\beta_{T} \varepsilon_{y}^{\prime}\right), \\
& g=2\left(b \gamma_{T}-d \alpha_{T}\right) \varepsilon_{x}+2\left(b \delta_{T}-d \beta_{T}\right) \varepsilon_{x}^{\prime} \\
& h=2\left(b \gamma_{T}-d \alpha_{T}\right) \varepsilon_{y}+2\left(b \delta_{T}-d \beta_{T}\right) \varepsilon_{y}^{\prime}
\end{aligned}
$$

where $\varepsilon_{x}, \varepsilon_{x}^{\prime}, \varepsilon_{y}$ and $\varepsilon_{y}^{\prime}$ denote the two-dimensional misalignment parameters, $\varepsilon_{x}$ and $\varepsilon_{y}$ are the displacement elements in $x$ and $y$ direction respectively, $\varepsilon_{x}^{\prime}$ and $\varepsilon_{y}^{\prime}$ are the tilting angle of the elements in $x$ and $y$ direction respectively.
$\alpha_{T}, \beta_{T}, \gamma_{T}$ and $\delta_{T}$ represent the misaligned matrix elements determined by

$$
\begin{gathered}
\alpha_{T}=1-a, \beta_{T}=l-b, \\
\gamma_{T}=-c, \delta_{T}= \pm 1-d
\end{gathered}
$$

For forward-going optical elements, $\delta_{T}$ is chosen for "+"sign; for backward-going ones, $\delta_{T}$ is chosen for "-"sign. Eqs. (6) and (9) can be used conveniently to study the propagation of isotropic or anisotropic GSM beam with or without twist phase through paraxial aligned and misaligned ABCD optical systems. With these formulae, the focusing properties and spectral shift of GSM beam were investigated in [39-41].

\section{PROPAGATION OF A GSM BEAM THROUGH APERTURED OPTICAL SYSTEM}

Aperture is commonly encountered in practical optical system, and it has been found some interesting phenomena such as focal shift and focal switch [81, 82], spectral shift and spectral switch [83], are closely related to the aperture size. The typical method for treating the propagation of partially coherent light through an apertured optical system is the time-consuming numerical integration. Thus it is important and interesting to derive analytical propagation formulae for a GSM beam passing through apertured optical system.

Following [84], the hard aperture function of a circular aperture with radius $a_{1}$ can be expanded as the following finite sum of complex Gaussian functions

$$
H\left(\mathbf{r}_{1}\right)=\sum_{m=1}^{M} A_{m} \exp \left(-\frac{B_{m}}{a_{1}^{2}} \mathbf{r}_{1}^{2}\right)
$$

where $A_{m}$ and $B_{m}$ are the expansion and Gaussian coefficients, which can be obtained by optimization computation, a table of $A_{m}$ and $B_{m}$ can be found in Ref. [84]. After some arrangement, we can express $H\left(\boldsymbol{r}_{1}\right) H^{*}\left(\boldsymbol{r}_{2}\right)$ in following tensor form [42]

$$
H\left(\mathbf{r}_{1}\right) H^{*}\left(\mathbf{r}_{2}\right)=\sum_{m=1}^{M} \sum_{n=1}^{M} A_{m} A_{n}^{*} \exp \left[-\frac{i k}{2} \tilde{\mathbf{r}}^{T} \mathbf{B}_{m n} \tilde{\mathbf{r}}\right]
$$

where

$$
\mathbf{B}_{m n}=\frac{2}{i k a_{1}^{2}}\left(\begin{array}{cc}
B_{m} \mathbf{I} & 0 \mathbf{I} \\
0 \mathbf{I} & B_{n}^{*} \mathbf{I}
\end{array}\right)
$$

here "*” denotes the complex conjugate. Substituting Eqs. (3) and (19) into the generalized Collins formula, we obtain following analytical propagation formula for a GSM beam passing through an circularly apertured ABCD optical system [42] 


$$
\begin{aligned}
W(\tilde{\mathbf{n}})= & \sum_{m=1}^{M} \sum_{n=1}^{M} A_{m} A_{n}^{*}\left[\operatorname{det}\left(\tilde{\mathbf{A}}+\tilde{\mathbf{B}} \mathbf{M}_{1}^{-1}+\tilde{\mathbf{B}} \mathbf{B}_{m n}\right)\right]^{-1 / 2} \\
& \times \exp \left[-\frac{i k}{2} \tilde{\boldsymbol{\rho}}^{\mathrm{T}} \mathbf{M}_{2 m n}^{-1} \tilde{\boldsymbol{\rho}}\right]
\end{aligned}
$$

with

$$
\begin{aligned}
\mathbf{M}_{2 m n}^{-1}= & {\left[\tilde{\mathbf{C}}+\tilde{\mathbf{D}}\left(\mathbf{M}_{i}^{-1}+\mathbf{B}_{m n}\right)\right] } \\
& \times\left[\tilde{\mathbf{A}}+\tilde{\mathbf{B}}\left(\mathbf{M}_{1}^{-1}+\mathbf{B}_{m n}\right)\right]^{-1}
\end{aligned}
$$

In a similar way, we obtain following analytical propagation formula for a GSM beam passing through a rectangulary apertured $\mathrm{ABCD}$ optical system [42]

$$
\begin{aligned}
\mathrm{W}(\tilde{\mathbf{n}}) & =G_{0} \sum_{m=1}^{M} \sum_{n=1}^{N} \sum_{p=1}^{M} \sum_{l=1}^{N} A_{m} A_{n} A_{p}^{*} A_{l}^{*} \\
& \times\left[\operatorname{det}\left(\tilde{\mathbf{A}}+\tilde{\mathbf{B}} \mathbf{M}_{1}^{-1}+\tilde{\mathbf{B}} \mathbf{B}_{m n p l}\right)\right]^{-1 / 2} \\
& \times \exp \left[-\frac{i k}{2} \tilde{\boldsymbol{\rho}}^{\mathrm{T}} \mathbf{M}_{2 m n p l}^{-1} \tilde{\boldsymbol{\rho}}\right]
\end{aligned}
$$

Where

$$
\mathbf{B}_{m n p l}=\frac{2}{i k}\left(\begin{array}{cccc}
B_{m} / a_{1}^{2} & 0 & 0 & 0 \\
0 & B_{n} / b_{1}^{2} & 0 & 0 \\
0 & 0 & B_{p}^{*} / a_{1}^{2} & 0 \\
0 & 0 & 0 & B_{l}^{*} / b_{1}^{2}
\end{array}\right) \text {, }
$$

$$
\begin{aligned}
& \mathbf{M}_{2 m n p l}^{-1}=\left[\tilde{\mathbf{C}}+\tilde{\mathbf{D}}\left(\mathbf{M}_{1}^{-1}+\mathbf{B}_{m n p l}\right)\right] \\
& {\left[\tilde{\mathbf{A}}+\tilde{\mathbf{B}}\left(\mathbf{M}_{1}^{-1}+\mathbf{B}_{m n p l}\right)\right]^{-1} }
\end{aligned}
$$

with $2 a_{1}$ and $2 b_{1}$ being the aperture widths in $x$ - and $y$ directions. Under the condition of $a_{1} \rightarrow \infty$ and $b_{1} \rightarrow \infty$, Eq. (21) or (23) reduces to Eq. (6), and Eq. (22) or (25) reduces to $\mathrm{Eq} .(7)$.

As a numerical example, we calculate in Fig. (1) the normalized intensity (cross line, $y=0$ ) of a twisted anisotropic GSM beam at $\mathrm{z}=60 \mathrm{~mm}$ after passing through a square aperture $\left(a_{1}=b_{1}\right)$ in front of a thin lens with focal length $f=50 \mathrm{~mm}$ (located at $\mathrm{z}=0$ ) versus different values of aperture widths by using analytical formula (solid line) and by direct numerically integration (dots). We choose the parameters as

$$
\begin{gathered}
\lambda=632.8 \mathrm{~nm},\left(\hat{o}_{I}^{2}\right)^{-1}=\mathbf{I}(\mathrm{mm})^{-2},\left(o_{g}^{2}\right)^{-1}=4 \mathbf{I}(\mathrm{mm})^{-2}, \\
\mathbf{R}^{-1}=0 \mathbf{I}, \quad \mu=0.00001, \quad \mathbf{A}=(1-z / f) \mathbf{I}, \mathbf{B}=z \mathbf{I},
\end{gathered}
$$

$\mathbf{C}=(-1 / f) \mathbf{I}, \mathbf{D}=\mathbf{I}, A_{i}$ and $B_{i}$ are chosen to be the same as those in Ref. [84] with $M=N=10$. From Fig. (1), we can find that the simulation results calculated by analytical formula are in a good agreement with those obtained by numerical integration, except for some slight deviations as aperture widths increase. Such deviations increases dramatically when $M$ and $N$ decrease from 10 to a smaller value, while almost don't change when $M$ and $N$ increase from 10 to a larger value, thus $M=N=10$ is necessary and

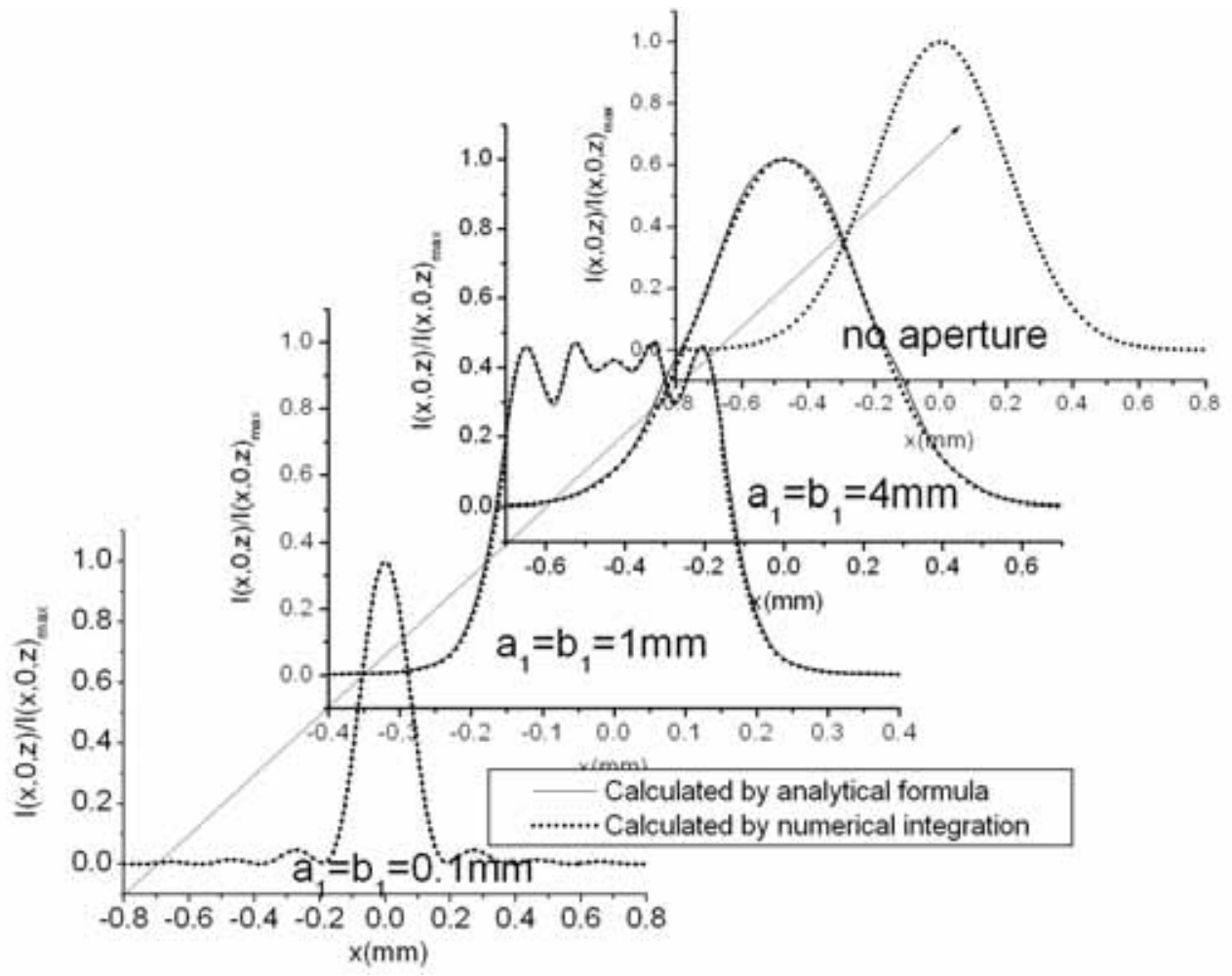

Fig. (1). Normalized intensity (cross line, $\mathrm{y}=0$ ) of a twisted anisotropic GSM beam at $\mathrm{z}=60 \mathrm{~mm}$ versus different values of aperture widths [42]. 
sufficient. Our analytical formula is very fast for calculation while the direct numerical integration is very timeconsuming. It takes $75 \mathrm{~s}$ to calculate the solid lines by analytical formula, while it takes over 20 hours to calculate the dots by numerical integration using the algorithm named successive adaptive Simpson rule with the truncation error assumed to be $10^{-6}$. One can also see from Fig. (1) that the influence of the aperture on the irradiance distribution gradually disappears as aperture widths increase. In conclusion, the analytical propagation formula avoids timeconsuming numerical integration and provides a convenient and effective way for studying the propagation and transformation of a partially coherent GSM beam through an apertured paraxial optical system.

\section{FRACTIONAL FOURIER TRANSFORM FOR A GSM BEAM: THEORY}

The fractional Fourier transform (FRT) is the generalization of the conventional Fourier transform, which was first introduced into optics by Ozaktas, Mendlovic and Lohmann in 1993 [85, 86], and has been extensively studied due to its important applications in signal processing, optical image encryption, beam shaping and beam analysis $[87,88]$. In this section, we introduced the tensor method to treat the FRT for a GSM beam.

The two types of optical system for performing the FRT are shown in Fig. (2). Assume a stationary polychromatic source field $E(\mathbf{r})$. The FRT achieved by a thin lens system under paraxial approximation performs the following operation [86]:

$$
\begin{array}{r}
E_{p}(\mathbf{u})=\frac{1}{i \lambda f \sin \phi} \int_{-\infty}^{\infty} E(\mathbf{r}) \exp \left[-\frac{i \pi\left(|\mathbf{r}|^{2}+|\mathbf{u}|^{2}\right)}{\lambda f \tan \phi}\right] \\
\exp \left[\frac{2 \pi i \mathbf{r} \cdot \mathbf{u}}{\lambda f \sin \phi}\right] d^{2} \mathbf{r}
\end{array}
$$

where $\mathbf{r}$ and $\mathbf{u}$ are the position vectors in the input plane and the FRT plane, respectively. $\lambda$ is the optical wavelength, $f$ is the standard focal length. The fractional order of the FRT is given by $p=2 \phi / \pi$. When $p=1$, the FRT goes back to the conventional Fourier transform. In Eq. (26), a factor $1 /(i \lambda f \sin \phi)$ existed in front of the integral ensures the energy conservation after FRT transform.

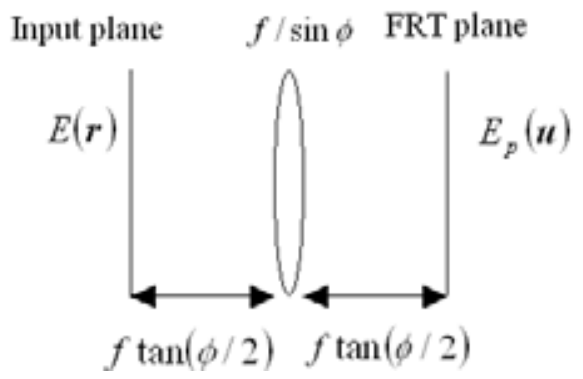

Assume the optical fields at the two arbitrary points $\mathbf{r}_{1}, \mathbf{r}_{2}$ in the input plane are $E\left(\mathbf{r}_{1}\right), E\left(\mathbf{r}_{2}\right)$, the optical fields at the two arbitrary points $\mathbf{u}_{1}, \mathbf{u}_{2}$ in the FRT plane are $E_{p}\left(\mathbf{u}_{1}\right), E_{p}\left(\mathbf{u}_{2}\right)$, respectively. The cross-spectral density in the input plane $\mathrm{W}(\tilde{\mathbf{r}})$, and that in the FRT plane $\mathrm{W}_{p}(\tilde{\mathbf{u}})$ are defined by:

$$
\mathrm{W}(\tilde{\mathbf{r}})=\left\langle E\left(\mathbf{r}_{1}\right) E^{*}\left(\mathbf{r}_{2}\right)\right\rangle, \mathrm{W}_{p}(\tilde{\mathbf{u}})=\left\langle E_{p}\left(\mathbf{u}_{1}\right) E_{p}^{*}\left(\mathbf{u}_{2}\right)\right\rangle,
$$

where the angular bracket "<>" denotes an ensemble average, $\tilde{\mathbf{r}}^{T}=\left(\begin{array}{ll}\mathbf{r}_{1}^{T} & \mathbf{r}_{2}^{T}\end{array}\right), \tilde{\mathbf{u}}^{T}=\left(\begin{array}{ll}\mathbf{u}_{1}^{T} & \mathbf{u}_{2}^{T}\end{array}\right)$. Substituting Eq. (26) into Eq. (27), we can get the relation between the cross-spectral density of partially coherent beam in the FRT plane and in the input plane as follows:

$$
\begin{aligned}
& \mathrm{W}_{p}(\tilde{\mathbf{u}})= \frac{1}{(\lambda f \sin \phi)^{2}} \iiint \int W_{0}(\tilde{\mathbf{r}}) \\
& \exp \left[-\frac{i \pi\left(\left|\mathbf{r}_{1}\right|^{2}+\left|\mathbf{u}_{1}\right|^{2}-\left|\mathbf{r}_{2}\right|^{2}-\left|\mathbf{u}_{2}\right|^{2}\right)}{\lambda f \tan \phi}\right] \\
& \quad \times \exp \left[\frac{2 \pi i\left(\mathbf{r}_{1} \cdot \mathbf{u}_{1}-\mathbf{r}_{2} \cdot \mathbf{u}_{2}\right)}{\lambda f \sin \phi}\right] d \mathbf{r}_{1} d \mathbf{r}_{2},
\end{aligned}
$$

After some arrangement, Eq. (28) can be expressed in following alternative tensor form [43]

$$
\begin{aligned}
& \mathrm{W}_{p}(\tilde{\mathbf{u}})=\frac{k^{2}}{4 \pi^{2}[\operatorname{det}(\overline{\mathbf{B}})]^{1 / 2}} \\
& \iiint \int \Gamma(\tilde{\mathbf{r}}) \exp \left[-\frac{i k}{2}\left(\begin{array}{r}
\tilde{\mathbf{r}}^{T} \overline{\mathbf{B}}^{-1} \overline{\mathbf{A}} \tilde{\mathbf{r}}-2 \tilde{\mathbf{r}}^{T} \overline{\mathbf{B}}^{-1} \tilde{\mathbf{u}} \\
+\tilde{\mathbf{u}}^{T} \overline{\mathbf{D}} \overline{\mathbf{B}}^{-1} \tilde{\mathbf{u}}
\end{array}\right)\right] d \tilde{\mathbf{r}},
\end{aligned}
$$

with

$$
\begin{aligned}
& \overline{\mathbf{A}}=\cos \phi\left(\begin{array}{cc}
\mathbf{I} & 0 \mathbf{I} \\
0 \mathbf{I} & \mathbf{I}
\end{array}\right), \overline{\mathbf{B}}=f \sin \phi\left(\begin{array}{cc}
\mathbf{I} & \mathbf{0} \\
\mathbf{0} & -\mathbf{I}
\end{array}\right), \\
& \overline{\mathbf{C}}=\frac{\sin \phi}{f}\left[\begin{array}{cc}
-\mathbf{I} & \mathbf{0} \\
\mathbf{0} & \mathbf{I}
\end{array}\right], \overline{\mathbf{D}}=\cos \phi\left(\begin{array}{cc}
\mathbf{I} & \mathbf{0} \\
\mathbf{0} & \mathbf{I}
\end{array}\right)
\end{aligned}
$$

Substituting Eq. (3) into Eq. (29), we obtain (after some vector integration and tensor operation) [43]

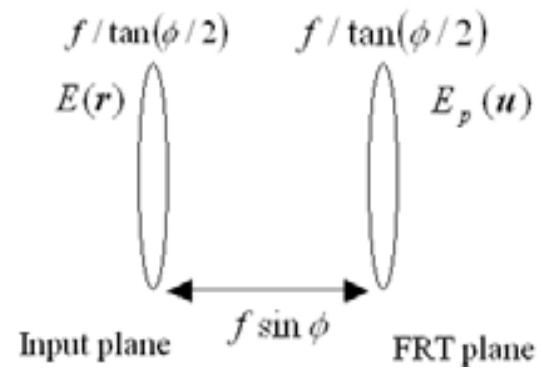

Fig. (2). Optical system for performing the FRT. (a) one lens system, (b) two lenses system [86]. 


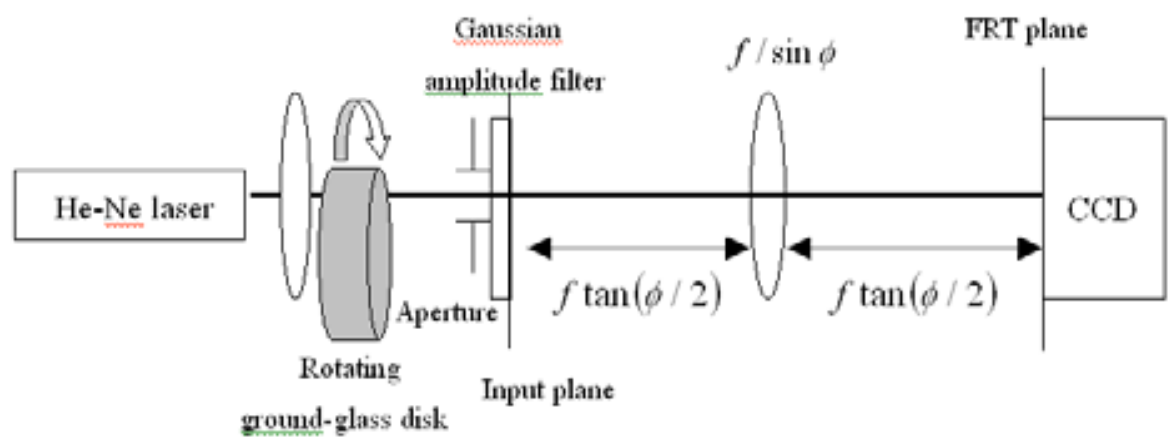

Fig. (3). Experimental setup for observing the FRT for a GSM beam [90].

$\mathrm{W}_{p}(\tilde{\mathbf{u}})=\frac{G_{0}}{\left[\operatorname{det}\left(\overline{\mathbf{A}}+\overline{\mathbf{B}} \mathbf{M}_{1}^{-1}\right)\right]^{1 / 2}} \exp \left(-\frac{i k}{2} \tilde{\mathbf{u}}^{T} \mathbf{M}_{2}^{-1} \tilde{\mathbf{u}}\right)$,

$\mathbf{M}_{2}^{-1}$ is related with $\mathbf{M}_{1}^{-1}$ by

$\mathbf{M}_{2}^{-1}=\left(\overline{\mathbf{C}}+\overline{\mathbf{D}} \mathbf{M}_{1}^{-1}\right)\left(\overline{\mathbf{A}}+\overline{\mathbf{B}} \mathbf{M}_{1}^{-1}\right)^{-1}$,

Eq. (31) is the analytical propagation formula for a GSM beam passing through the FRT optical system, and Eq. (32) is the equivalent tensor $\mathrm{ABCD}$ law for a GSM beam performing a FRT.

\section{FRACTIONAL FOURIER TRANSFORM FOR A GSM BEAM: EXPERIMENT}

Now we introduced the experimental observation of FRT for a isotropic GSM beam in this section. Fig. (3) shows our experimental setup for realizing the FRT for a GSM beam. A focused laser beam generated by a $\mathrm{He}-\mathrm{Ne}$ laser $(\lambda=632.8 \mathrm{~nm})$ is used to illuminate a rotating ground-glass disk, and the transmitted light can be considered as a partially coherent light with Gaussian statistics. A small circular aperture (diameter $d \approx 1.2 \mathrm{~cm}$ ) located behind ground-glass disk is used to select a portion of the scattered light, and a Gaussian amplitude filter located behind the aperture is used to transform the intensity distribution of the partially coherent light into Gaussian distribution. The transmitted beam behind the Gaussian amplitude filter can be approximately regarded as a isotropic GSM beam, and we take the transmitted light just behind the Gaussian amplitude filter as the light source in the input plane.

The intensity distributions in the source plane and the FRT plane are measured directly by a CCD (DXM1200F), and the corresponding software for CCD is supplied by Nikon corporation. Fig. (4) shows our experimental results of the intensity distribution and the corresponding normalized intensity distribution at cross line $\mathrm{y}=0$ in the input plane. In Fig. (4b), the dotted line is the experimental result and the solid line is the result of Gaussian fit for the experimental data. One can see from Fig. (4) that the intensity distribution in the input plane agrees well with the Gaussian distribution and the transverse spot width $\sigma_{I 0} \approx 2 \mathrm{~mm}$. We use the coincidence optical system similar to Fig. (3) of Ref. [89] to measure the spectral degree of coherence of partially coherent beam. The detailed principle

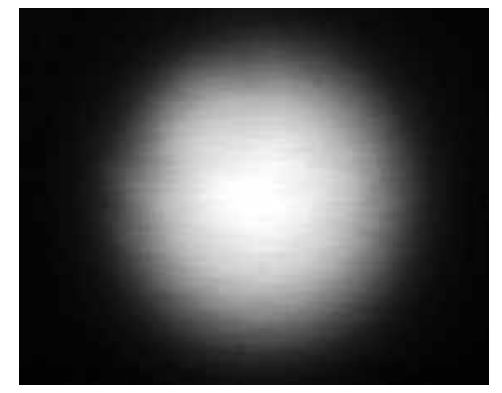

(a)

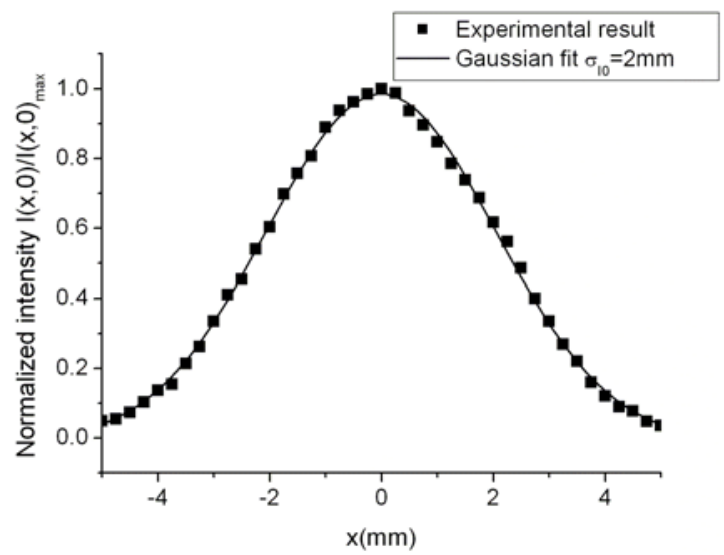

(b)

Fig. (4). Experimental results of (a) the intensity distribution, and (b) the corresponding normalized intensity distribution at cross line $\mathrm{y}=0$ in the input plane (dotted line). The solid line is a result of Gaussian fit [90].

for measuring the spectral degree of coherence can be found in Ref. [89]. Fig. (5) shows our experimental result (dotted line) of the modulus of the square of the spectral degree of coherence $\left|g^{2}\left(x_{1}-x_{2}\right)\right|$ (along $\left.x_{1}-x_{2}\right)$ for the partially coherent beam in the input plane and the corresponding result of the Gaussian fit (solid line) for the experimental data. One sees from Fig. (5) that the spectral degree of coherence is also of Gaussian distribution and the coherence width $\sigma_{g 0} \approx 0.079 \mathrm{~mm}$. Thus, the partially coherent beam in the input plane can be approximately regarded as a isotropic GSM beam. By varying the focused beam spot on the ground-glass disk, we can control the coherence of the generated GSM beam in the input plane. 


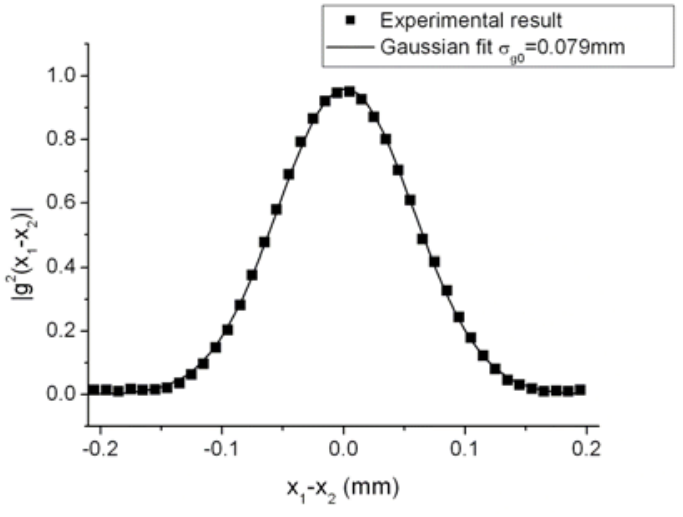

Fig. (5). Experimental result of the modulus of the square of the spectral degree of coherence $\left|g^{2}\left(x_{1}-x_{2}\right)\right|$ (along $\left.x_{1}-x_{2}\right)$ for the partially coherent beam in the input plane [90].
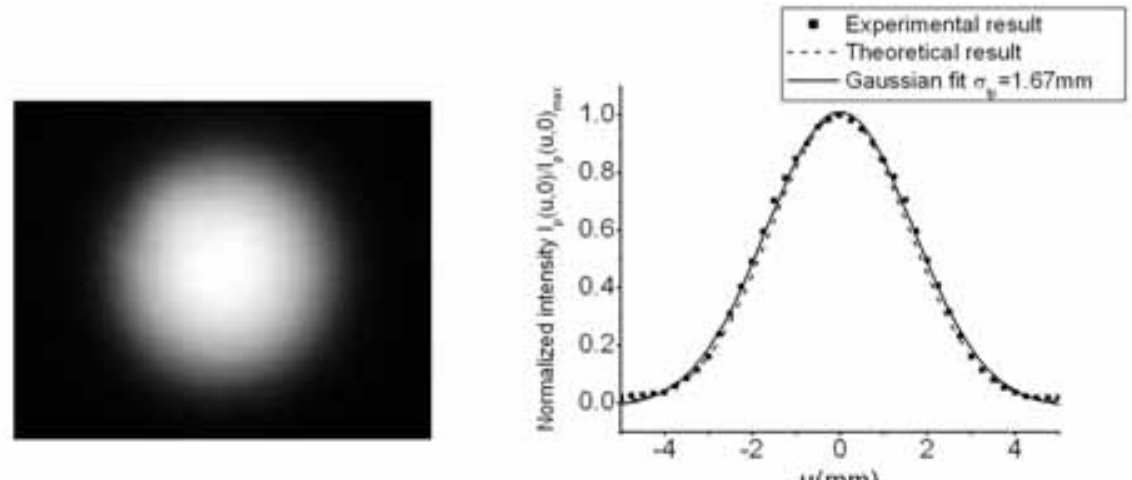

(a)
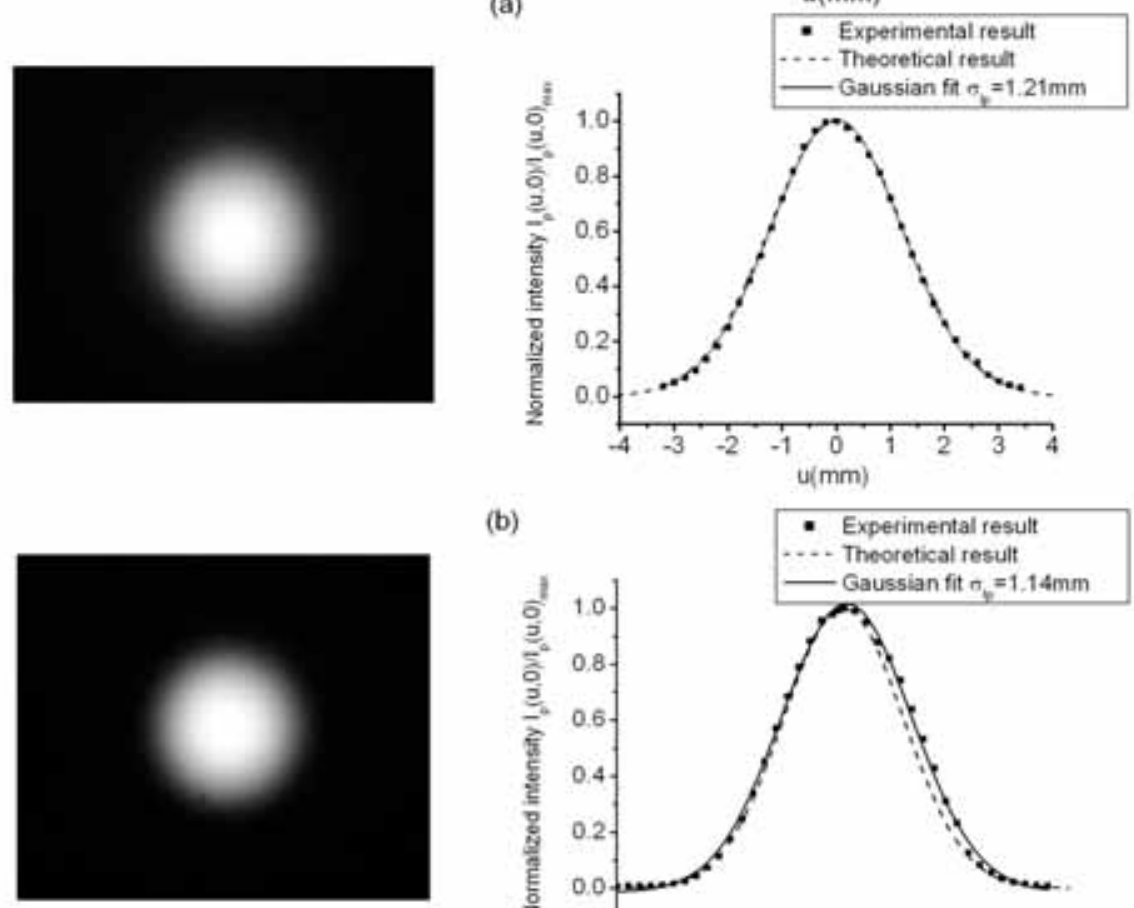

(b) distributions and the corresponding normalized intensity distributions (dotted lines) at cross line $v=0$ in the FRT plane for different fractional order $p$ with $f=40 \mathrm{~cm}$. For comparison, the corresponding theoretical results (dashed line) and curves of Gaussian fit for experimental results (solid line) are also shown in Fig. (6). Fig. (7) show the experimental results of the modulus of the square of the spectral degree of coherence $\left|g_{p}^{2}\left(u_{1}-u_{2}\right)\right|$ (along $u_{1}-u_{2}$ ) for the partially coherent beam in the FRT plane for different fractional order $p$. For comparison, the corresponding theoretical results (dashed line) and curves of Gaussian fit for experimental results (solid line) are also shown in Fig. (7). One finds from Figs. (6 and 7) that both intensity distribution and spectral degree of coherence in the FRT plane have Gaussian distributions. The beam width and coherence width depends on the fractional order $p$. The

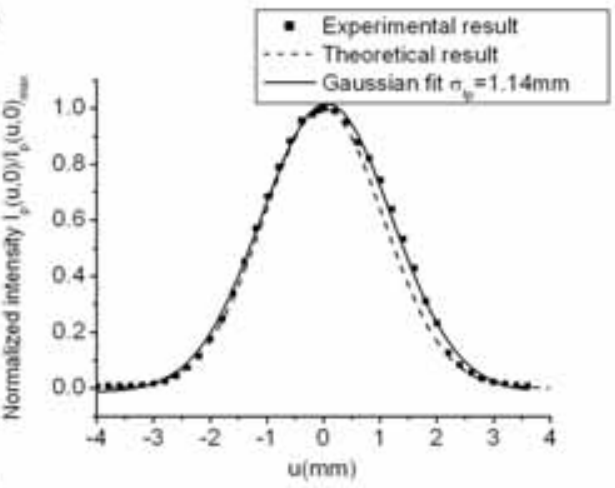

(c)

Fig. (6). Experimental results of the intensity distributions and the corresponding normalized intensity distributions (dotted lines) at cross line $v=0$ in the FRT plane for different fractional order $p$ (a) $p=0.44$ (b) $p=0.60$ (c) $p=0.68$. The solid lines are the corresponding results of Gaussian fit for the experimental results and the dashed lines are the corresponding theoretical results [90]. 


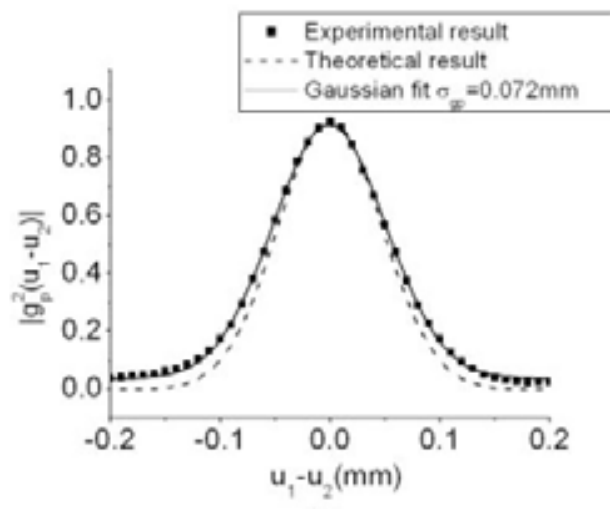

(a)

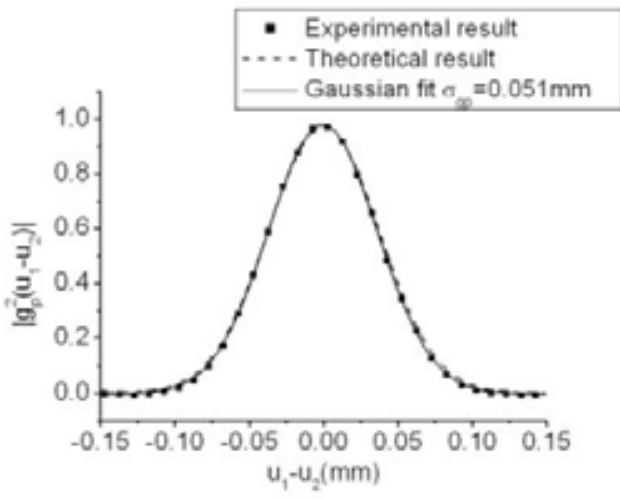

(b)

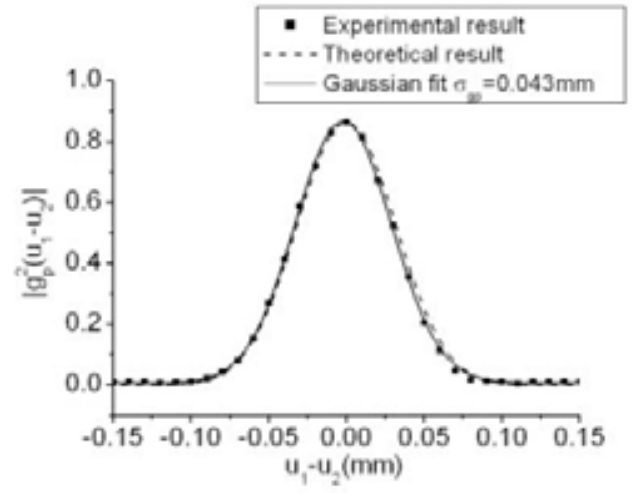

(c)

Fig. (7). Experimental results of the modulus of the square of the spectral degree of coherence $\left|g_{p}^{2}\left(u_{1}-u_{2}\right)\right|$ (along $u_{1}-u_{2}$ ) for the partially coherent beam in the FRT plane for different fractional order $p$ (a) $p=0.44$ (b) $p=0.60$ (c) $p=0.68$. The solid lines are the corresponding results of Gaussian fit for the experimental results and the dashed lines are the corresponding theoretical results [90].

experimental results also agree reasonably well with the theoretical results. Thus the tensor method and derived analytical formulae provide an effective and reliable way for analyzing the properties of a GSM beam in the FRT plane.

With the help of tensor method, we have also derived the analytical propagation formulae for a GSM beam passing through a truncated FRT optical system [91], and we have carried out experimental observation of the truncated FRT for a GSM beam. The experimental results are consistent with the theoretical results. Our results show that initial source coherence, fractional order, and aperture width (i.e., truncation parameter) have strong influences on the intensity and coherence properties of the partially coherent beam in the FRT plane. When the aperture width is large, both the intensity and the spectral degree of coherence in the FRT plane are of Gaussian distribution. As the aperture width decreases, the diffraction pattern gradually appears in the FRT plane, and the spectral degree of coherence becomes of non-Gaussian distribution. The truncated FRT optical system provides a flexible way to control the intensity distribution and coherence properties of partially coherent beam.

\section{PROPAGATION OF A GSM BEAM THROUGH DISPERSIVE AND ABSORBING MEDIA}

Supposing that light beam propagates through the media, which occupies the half space $z>0$. The diffraction integral formula for a beam passing through the media reads [92]:

$$
E\left(\tilde{\mathbf{n}}_{1}\right)=-\frac{i k}{2 \pi z} \iint E\left(\mathbf{r}_{1}\right) \exp (-i k l) d \mathbf{r}_{1}
$$

where $k=k_{0} n(\omega)=\omega n(\omega) / c$ is the wavenumber in the medium, $n(\omega)$ is the refractive index of the medium that is generally complex, $\mathrm{c}$ is the speed of light in vacuum, $k_{0}=\omega / c$ is the wavenumber in free space. $l$ is the Hamiltonian's point characteristic function (or called eikonal function) between the incident and output planes, given by [92]:

$l=l_{0}+\frac{1}{2 z}\left(\mathbf{r}_{1}^{2}-2 \mathbf{r}_{1} \cdot \tilde{\mathbf{n}}_{1}+\tilde{\mathbf{n}}_{1}^{2}\right)$,

here $l_{0}$ is the optical path along axis.

Using Eq. (33), we can get the generalized diffraction integral formula for the cross-spectral density of partially coherent beam in dispersive and absorbing media as follows [9]

$$
\begin{aligned}
\mathrm{W}\left(\tilde{\mathrm{n}}_{1}, \tilde{\mathrm{n}}_{2}\right)= & \frac{k k^{*}}{4 \pi^{2} z^{2}} \iiint \int \mathrm{W}\left(\mathbf{r}_{1}, \mathbf{r}_{2}\right) \exp \left[-\frac{i k}{2 z}\left(\mathbf{r}_{1}^{2}-2 \mathbf{r}_{1} \cdot \tilde{\mathrm{n}}_{1}+\tilde{\mathrm{n}}_{1}^{2}\right)\right] \\
& \times \exp \left[\frac{i k}{2 z}\left(\mathbf{r}_{2}^{2}-2 \mathbf{r}_{2} \cdot \tilde{\mathrm{n}}_{2}+\tilde{\mathrm{n}}_{2}^{2}\right)\right] d \mathbf{r}_{1} d \mathbf{r}_{2},
\end{aligned}
$$



form

Eq. (35) can be express in following alternative tensor

$$
\begin{aligned}
\mathrm{W}(\tilde{\mathbf{n}}) & =\frac{\exp \left(i k l_{0}-i k^{*} l_{0}\right)}{\pi^{2}[\operatorname{det}(\tilde{\mathbf{B}})]^{1 / 2}} \iiint \int W(\tilde{\mathbf{r}}) \\
& \times \exp \left(-\tilde{\mathbf{r}}^{T} \tilde{\mathbf{B}}^{-1} \tilde{\mathbf{r}}-2 \tilde{\mathbf{r}}^{T} \tilde{\mathbf{B}}^{-1} \tilde{\mathbf{n}}+\tilde{\mathbf{n}}^{T} \tilde{\mathbf{B}}^{-1} \tilde{\mathbf{n}}\right) d \tilde{\mathbf{r}}
\end{aligned}
$$

For the convenience of integration, we express Eq. (1) in following alternative tensor form

$\mathrm{W}(\tilde{\mathbf{r}})=G_{0} \exp \left(-\tilde{\mathbf{r}}^{T} \mathbf{M}_{1}^{-1} \tilde{\mathbf{r}}\right)$,

where $\tilde{\boldsymbol{M}}_{1}^{-1}$ is expressed as

$$
\tilde{\mathbf{M}}_{1}^{-1}=\left(\begin{array}{cc}
\frac{i \omega}{2 c} \mathbf{R}^{-1}+\frac{1}{4}\left(\hat{o}_{I}^{2}\right)^{-1}+\frac{1}{2}\left(\hat{o}_{g}^{2}\right)^{-1} & -\frac{1}{2}\left(\hat{o}_{g}^{2}\right)^{-1}+\frac{i \omega}{2 c} \mu \mathbf{J} \\
-\frac{1}{2}\left(\hat{o}_{g}^{2}\right)^{-1}+\frac{i \omega}{2 c} \mu \mathbf{J}^{T} & -\frac{i k}{2} \mathbf{R}^{-1}+\frac{1}{4}\left(\hat{o}_{I}^{2}\right)^{-1}+\frac{1}{2}\left(\hat{o}_{g}^{2}\right)^{-1}
\end{array}\right)
$$

Substituting Eq. (37) into Eq. (35), we obtain (after vector integration and tensor operation) [45]

$$
W(\tilde{\mathbf{n}})=\frac{G_{0} \exp \left(i k l_{0}-i k^{*} l_{0}\right)}{\left[\operatorname{det}\left(\tilde{\mathbf{I}}+\tilde{\mathbf{B}} \tilde{\mathbf{M}}_{1}^{-1}\right)\right]^{1 / 2}} \exp \left[-\tilde{\mathbf{n}}^{T}\left(\tilde{\mathbf{M}}_{1}+\tilde{\mathbf{B}}\right)^{-1} \tilde{\mathbf{n}}\right]
$$

where $\tilde{\mathbf{I}}$ is a $4 \times 4$ unit matrix. Eq. (39) can be used conveniently to analyze the intensity, coherence and spectrum properties of a GSM beam propagating in dispersive and absorbing media.

\section{PROPAGATION OF A GSM BEAM IN TURBULENT ATMOSPHERE}

Propagation characteristics of different types of beams propagating in the turbulent atmosphere are of interest for optical communications, imaging and remote sensing applications $[92,93]$. Within the framework of paraxial approximation, the propagation of the cross-spectral density of a partially coherent laser beam in a turbulent atmosphere can be treated with the following extended Huygens-Fresnel integral formula $[92,93]$

$$
\begin{aligned}
\mathrm{W}\left(\tilde{\mathrm{n}}_{1}, \tilde{\mathrm{n}}_{2}, z\right)= & \frac{k^{2}}{4 \pi^{2} z^{2}} \int_{-\infty}^{\infty} \int_{-\infty}^{\infty} \int_{-\infty}^{\infty} \int_{-\infty}^{\infty} W_{0}\left(\mathbf{r}_{1}, \mathbf{r}_{2}, 0\right) \\
& \times \exp \left[-\frac{i k}{2 z}\left(\mathbf{r}_{1}-\tilde{\mathrm{n}}_{1}\right)^{2}+\frac{i k}{2 z}\left(\mathbf{r}_{2}-\tilde{\mathrm{n}}_{1}\right)^{2}\right] \\
& \times\left\langle\exp \left[\Psi\left(\mathbf{r}_{1}, \tilde{\mathrm{n}}_{1}\right)+\Psi^{*}\left(\mathbf{r}_{2}, \tilde{\mathrm{n}}_{2}\right)\right]\right\rangle d \mathbf{r}_{1} d \mathbf{r}_{2},
\end{aligned}
$$

where < > denotes ensemble average over the turbulent medium. The ensemble average term in Eq. (40) can be expressed as [92, 93]

$$
\begin{aligned}
& \left\langle\exp \left[\Psi\left(\mathbf{r}_{1}, \tilde{\mathrm{n}}_{1}\right)+\Psi^{*}\left(\mathbf{r}_{2}, \tilde{\mathrm{n}}_{2}\right)\right]\right\rangle= \\
& \exp \left[-\frac{\left(\mathbf{r}_{1}-\mathbf{r}_{2}\right)^{2}}{\rho_{0}^{2}}-\frac{\left(\mathbf{r}_{1}-\mathbf{r}_{2}\right)\left(\tilde{\mathrm{n}}_{1}-\tilde{\mathrm{n}}_{2}\right)}{\rho_{0}^{2}}-\frac{\left(\tilde{\mathrm{n}}_{1}-\tilde{\mathrm{n}}_{2}\right)^{2}}{\rho_{0}^{2}}\right],
\end{aligned}
$$

where $\rho_{0}=\left(0.545 C_{n}^{2} k^{2} z\right)^{-3 / 5}$ is the coherence length of a spherical wave propagating in the turbulent medium with $C_{n}^{2}$ being the structure constant. In the derivation of Eq. (41), we have employed Kolmogorov spectrum and quadratic approximation for Rytov's phase structure function [92, 93].

Eq. (40) can be expressed in following alternative tensor form $[46,94]$

$$
\begin{aligned}
& \mathrm{W}(\tilde{\mathrm{n}}, z)=\frac{k^{2}}{4 \pi^{2}[\operatorname{det}(\tilde{\mathrm{n}})]^{1 / 2}} \int_{-\infty}^{\infty} \int_{-\infty}^{\infty} \int_{-\infty}^{\infty} \int_{-\infty}^{\infty} W_{0}(\tilde{\mathbf{r}}, 0) \\
& \times \exp \left[-\frac{i k}{2}\left(\tilde{\mathbf{r}}^{T} \tilde{\mathbf{B}}^{-1} \tilde{\mathbf{r}}-2 \tilde{\mathbf{r}} \tilde{\mathbf{B}}^{-1} \tilde{\mathbf{n}}+\tilde{\mathbf{n}}^{T} \tilde{\mathbf{B}}^{-1} \tilde{\mathbf{n}}\right)\right] \\
& \times \exp \left[-\frac{i k}{2} \tilde{\mathbf{r}}^{T} \tilde{\mathbf{P}} \tilde{\mathbf{r}}-\frac{i k}{2} \tilde{\mathbf{r}}^{T} \tilde{\mathbf{P}} \tilde{\mathrm{n}}-\frac{i k}{2} \tilde{\mathbf{n}}^{T} \tilde{\mathbf{P}} \tilde{\mathrm{n}}\right] d \tilde{\mathbf{r}},
\end{aligned}
$$

where $\tilde{\mathbf{B}}^{-1}$ and $\tilde{\mathbf{P}}$ are given by

$\tilde{\mathbf{B}}=\left(\begin{array}{cc}z \mathbf{I} & \mathbf{0} \\ \mathbf{0} & -z \mathbf{I}\end{array}\right), \quad \tilde{\mathbf{P}}=\frac{2}{i k \rho_{0}^{2}}\left(\begin{array}{cc}\mathbf{I} & -\mathbf{I} \\ -\mathbf{I} & \mathbf{I}\end{array}\right)$,

Substituting Eq. (3) into Eq. (42), we obtain (after vector integration) following analytical propagation formula for a GSM beam in turbulent atmosphere [46, 94]

$$
\mathrm{W}(\tilde{\mathbf{n}}, z)=\frac{1}{\left[\operatorname{det}\left(\tilde{\mathbf{I}}+\tilde{\mathbf{B}} \mathbf{M}_{1}^{-1}+\tilde{\mathbf{B}} \tilde{\mathbf{P}}_{1}\right)\right]^{1 / 2}} \exp \left[-\frac{i k}{2} \tilde{\mathbf{n}}^{T} \mathbf{M}_{2}^{-1} \tilde{\mathbf{n}}\right]
$$

where $\mathbf{M}_{2}^{-1}$ is the partially coherent complex curvature tensor in the receiver plane, and is related to $\mathbf{M}_{1}^{-1}$ by following relation

$$
\begin{aligned}
\mathbf{M}_{2}^{-1}= & \tilde{\mathbf{P}}_{1}+\tilde{\mathbf{B}}^{-1}-\left(\tilde{\mathbf{B}}^{-1}-\frac{1}{2} \tilde{\mathbf{P}}_{1}\right)^{T} \\
& \times\left(\mathbf{M}_{1}^{-1}+\tilde{\mathbf{B}}^{-1}+\tilde{\mathbf{P}}_{1}\right)^{-1}\left(\tilde{\mathbf{B}}^{-1}-\frac{1}{2} \tilde{\mathbf{P}}_{1}\right)
\end{aligned}
$$

The average intensity in the receiver plane is given by $\mathrm{I}(\tilde{\mathbf{n}}, z)=\mathrm{W}(\tilde{\mathbf{n}}, \tilde{\mathbf{n}}, z)$.

As a numerical example, we study the propagation properties of a twisted anisotropic GSM beam in turbulent atmosphere. We calculated in Fig. (8a-e) (contour graph) show the normalized irradiance distribution of a twisted anisotropic GSM beam $(\lambda=632.8 \mathrm{~nm})$ at several different propagation distances in a turbulent atmosphere. The initial parameters at $\mathrm{z}=0$ are set as $\boldsymbol{R}^{-1}=0, \mu=0.000005(\mathrm{~mm})$

For comparison, the far field irradiance distribution of a twisted anisotropic GSM beam in free space is also shown in Fig. (8f). From Fig. (a)-(c) one sees that in the near field the propagation of a twisted anisotropic AGSM beam is similar to that in free space, for example, the elliptical beam spot rotates clockwise (it will rotates anti-clockwise if $\mu<0$ ) gradually as $\mathrm{z}$ increases. While in the far field, the twisted anisotropic GSM beam eventually becomes a partially coherent stigmatic beam (see Fig. (8d-e) under the isotropic influence of the atmospheric turbulence on the beam profile, 


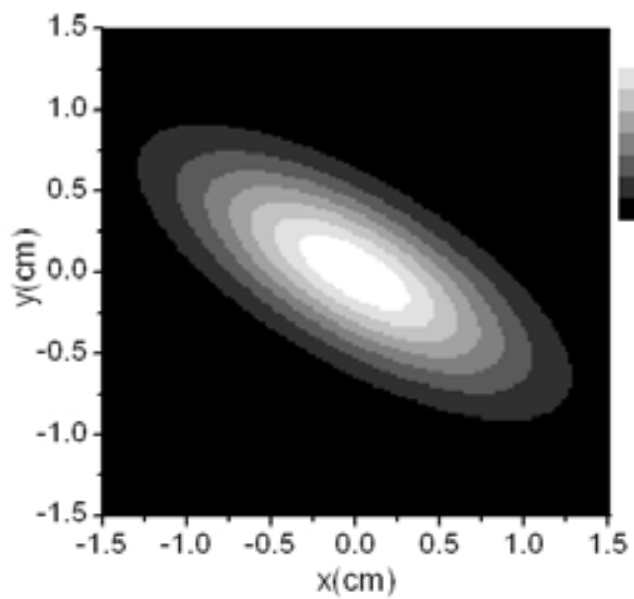

(a)

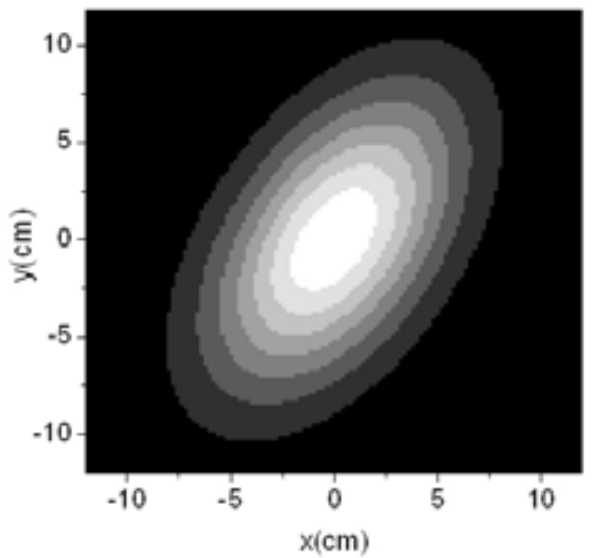

(c)

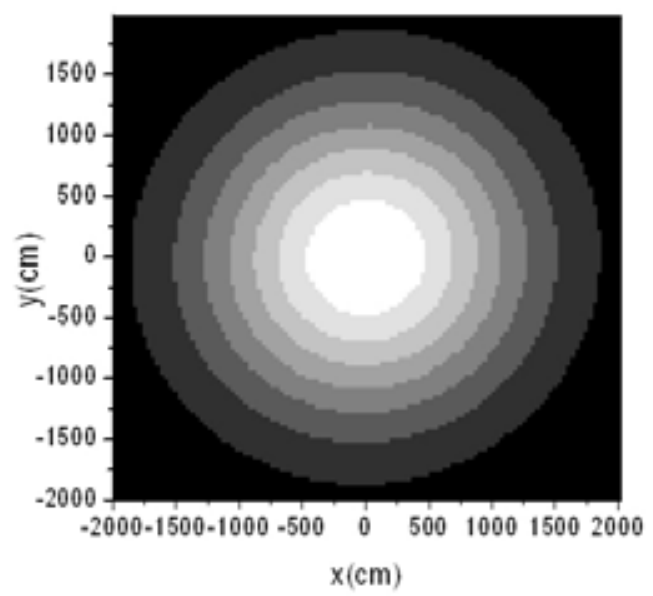

(e)

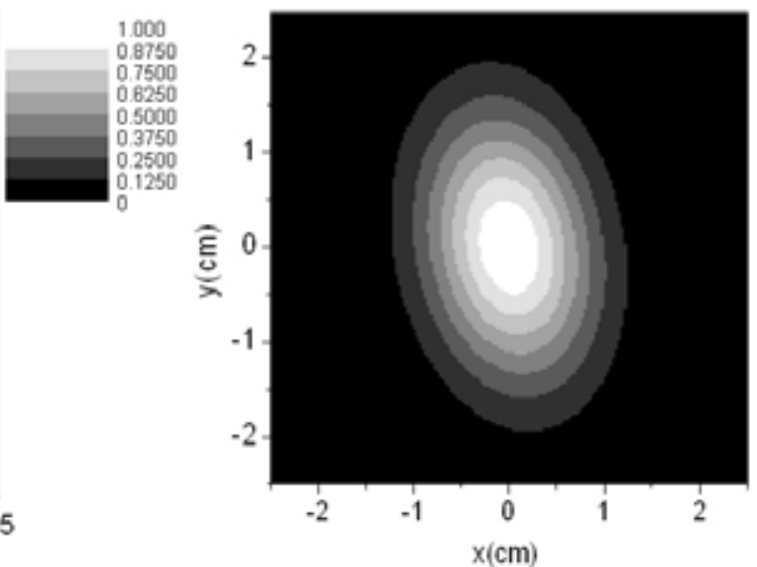

(b)

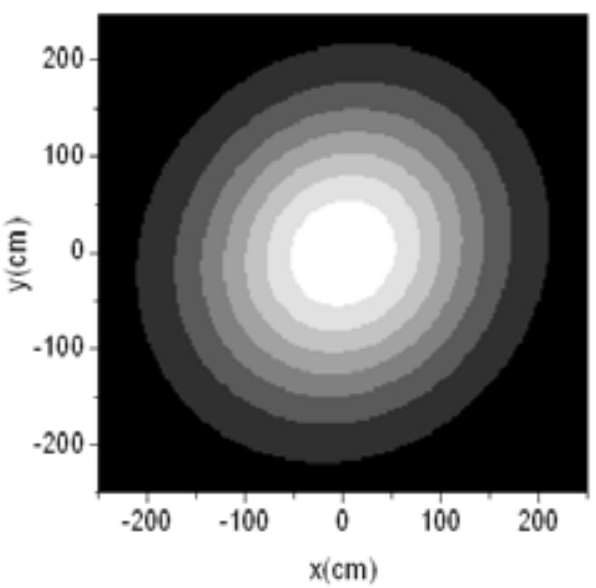

(d)

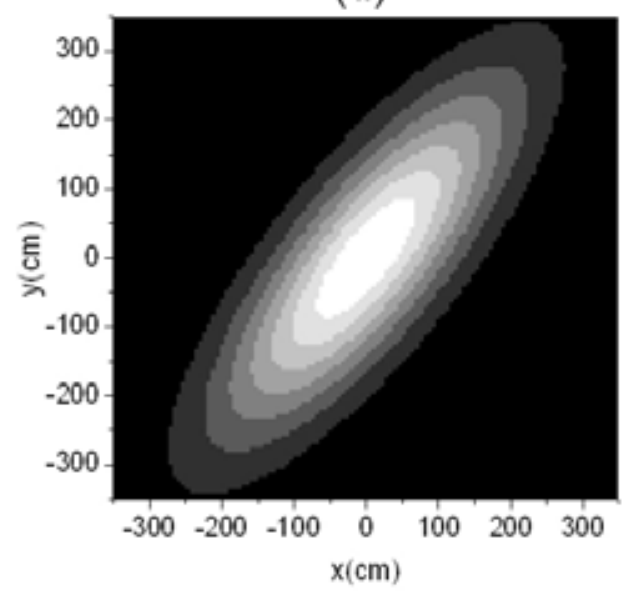

(f)

Fig. (8). Normalized irradiance distribution (contour graph) of a twisted anisotropic GSM beam at several different propagation distances in a turbulent atmosphere (with $C_{n}^{2}=10^{-13} \mathrm{~m}^{-2 / 3}$ ). (a) $\mathrm{z}=0 ;(\mathbf{b}) \mathrm{z}=0.15 \mathrm{~km} ;(\mathbf{c}) \mathrm{z}=1 \mathrm{~km} ;(\mathbf{d}) \mathrm{z}=10 \mathrm{~km} ;(\mathbf{e}) \mathrm{z}=40 \mathrm{~km} ;(\mathbf{f})$ the free space case (i.e., $C_{n}^{2}=0$ ) with $\mathrm{z}=40 \mathrm{~km}[46]$.

and then spreads as $\mathrm{z}$ increases further. These interesting propagation properties are quite different from those in free space. In free space, the beam spot remains elliptical in the far field (cf. Fig. ( $\mathbf{8} \mathbf{a}$ and $\mathbf{f})$ ). The propagation properties of a
TAGSM beam are closely related to the parameters of the beam and turbulent atmosphere. Numerical results in [46] clearly show tht the conversion of a twisted anisotropic GSM beam to a stigmatic beam in a turbulent atmosphere becomes 
slower as the coherence decreases and the absolute value of the twist factor increases. In other words, low coherence and larger twist have an effect of anti-circularization of the beam spot. The conversion of a twisted anisotropic GSM beam to a stigmatic beam also becomes quicker as the structure constant $C_{n}^{2}$ increases (i.e., stronger turbulence). Furthermore, the beam spot spreads more rapidly for a lower coherence width matrix, larger twist, or stronger turbulence.

$$
\begin{aligned}
& \mu=0.000005(\mathrm{~mm})^{-1}, C_{n}^{2}=10^{-13} m^{-2 / 3}, \\
& \left(\tilde{\mathrm{n}}_{I}^{2}\right)^{-1}=\left(\begin{array}{cc}
0.05 & 0.05 \\
0.05 & 0.1
\end{array}\right)(\mathrm{mm})^{-2},\left(\dot{o}_{g}^{2}\right)^{-1}=0.05 \mathrm{I}(\mathrm{mm})^{-2} .
\end{aligned}
$$

With the help of tensor method, the propagation properties of off-axis GSM beams, coherent partially coherent laser array beams, astigmatic Gaussian beams, flat-topped beam and dark hollow beams have also been investigated [94-99].

\section{PROPAGATION OF AN ELECTROMAGNETIC GSM BEAM THROUGH PARAXIAL OPTICAL SYSTEM IN FREE SPACE}

The second-order statistical properties of the electromagnetic GSM beam can be characterized by the $2 \times 2$ crossspectral density matrix $\overrightarrow{\mathrm{W}}\left(\mathbf{r}_{1}, \mathbf{r}_{2}, 0\right)$ specified at any two points with position vectors $\mathbf{r}_{1}$ and $\mathbf{r}_{2}$ in the source plane with elements [53-58]

$W_{\alpha \beta}\left(\mathbf{r}_{1}, \mathbf{r}_{2}\right)=A_{\alpha} A_{\beta} B_{\alpha \beta} \exp \left[-\frac{\mathbf{r}_{1}^{2}}{4 \sigma_{a}^{2}}-\frac{\mathbf{r}_{2}^{2}}{4 \sigma_{\beta}^{2}}-\frac{\left(\mathbf{r}_{1}-\mathbf{r}_{2}\right)^{2}}{2 \delta_{\alpha \beta}^{2}}\right]$,

$(\alpha=x, y ; \beta=x, y)$,

where $\mathbf{r}_{1}$ and $\mathbf{r}_{2}$ are the transverse position vectors (on the input plane), $\sigma_{\alpha}$ is the r.m.s width of the spectral density along $\alpha$ direction, $\delta_{x x}, \delta_{y y}$ and $\delta_{x y}$ are the r.m.s widths of auto-correlation functions of the $x$ component of the field, of the $y$ component of the field and of the mutual correlation function of $x$ and $y$ field components, respectively, $B_{x y}$ is the complex correlation coefficient between the $x$ and $y$ components of the electric field; Parameters $A_{\alpha}$, $B_{\alpha \beta}=\left|B_{\alpha \beta}\right| \exp \left(i \phi_{\alpha \beta}\right)=B_{\beta \alpha}^{*}, \quad \sigma_{\alpha}$ and $\delta_{\alpha \beta}$ are independent of position and, in our analysis, of frequency. The nine real parameters $A_{x}, A_{y}, \sigma_{x}, \sigma_{y},\left|B_{x y}\right|, \phi_{x y}, \delta_{x x}, \delta_{y y}$ and $\delta_{x y}$ entering the general model are shown to satisfy several intrinsic constraints and obey some simplifying assumptions (e.g. the phase difference between the $\mathrm{x}$ - an y-components of the field is removable, i.e. $\phi_{\alpha \alpha}=0$ ) $[58,59]$.

After some arrangement, Eq. (46) can be expressed in following tensor form [72-80]

$$
\begin{array}{r}
W_{\alpha \beta}(\tilde{\mathbf{r}})=A_{\alpha} A_{\beta} B_{\alpha \beta} \exp \left[-\frac{i k}{2} \tilde{\mathbf{r}}^{T} \mathbf{M}_{0 \alpha \beta}^{-1} \tilde{\mathbf{r}}\right], \\
(\alpha=x, y ; \beta=x, y)
\end{array}
$$

where the $4 \times 4$ matrix $\mathbf{M}_{0 \alpha \beta}^{-1}$ is expressed as follows

$$
\mathbf{M}_{0 \alpha \beta}^{-1}=\left(\begin{array}{cc}
\frac{1}{i k}\left(\frac{1}{2 \sigma_{a}^{2}}+\frac{1}{\delta_{\alpha \beta}^{2}}\right) \mathbf{I} & \frac{i}{k \delta_{\alpha \beta}^{2}} \mathbf{I} \\
\frac{i}{k \delta_{\alpha \beta}^{2}} \mathbf{I} & \frac{1}{i k}\left(\frac{1}{2 \sigma_{\beta}^{2}}+\frac{1}{\delta_{\alpha \beta}^{2}}\right) \mathbf{I}
\end{array}\right),
$$

After propagating through a general astigmatic $\mathrm{ABCD}$ optical system in free space, the element of the cross-spectral density matrix $\overrightarrow{\mathrm{W}}\left(\mathbf{r}_{1}, \mathbf{r}_{2}\right)$ can be expressed in the following tensor form [72-80]

$$
\begin{array}{r}
W_{\alpha \beta}(\tilde{\mathbf{n}})=A_{\alpha} A_{\beta} B_{\alpha \beta}\left[\operatorname{det}\left(\tilde{\mathbf{A}}+\tilde{\mathbf{B}} \mathbf{M}_{0 \alpha \beta}^{-1}\right)\right] \\
\times \exp \left[-\frac{i k}{2} \tilde{\mathbf{n}}^{T} \mathbf{M}_{1 \alpha \beta}^{-1} \tilde{\mathrm{n}}\right] \\
(\alpha=x, y ; \beta=x, y)
\end{array}
$$

where $\mathbf{M}^{-1}$ and $\mathbf{M}^{-1}$ are related by the following known tensor ABCD law [37]

$$
\mathbf{M}_{1 \alpha \beta}^{-1}=\left(\tilde{\mathbf{C}}+\tilde{\mathbf{D}} \mathbf{M}_{0 \alpha \beta}^{-1}\right)\left(\tilde{\mathbf{A}}+\tilde{\mathbf{B}} \mathbf{M}_{0 \alpha \beta}^{-1}\right)^{-1}
$$

In a similar way, with the help of tensor method, we can easily obtained the propagation formula for an electromagnetic GSM beam through a misaligned ABCD optical system in free space [73].

\section{EVOLUTION PROPERTIES OF AN ELECTROMAG- NETIC GSM BEAM IN A GAUSSIAN CAVITY}

Now we study the evolution properties of an electromagnetic GSM beam in a Gaussian cavity by applying Eqs. (49) and (50). For the sake of convenience, we will confine our analysis to a bare Gaussian cavity of length $L$ [see Fig. (9a)]. Here $M_{1}$ and $M_{2}$ are spherical mirrors each with radius of curvature $\mathrm{R}$ and Gaussian reflectivity profile with radius $\varepsilon$. Such resonator is equivalent to a sequence of identical thin spherical lenses with focal length $f=R / 2$, followed by the amplitude filters with a Gaussian transmission function [see Fig. (9b) for the equivalent (unfolded) optical system] [100]. The distance between each lens-filter pair is equal to $L$.

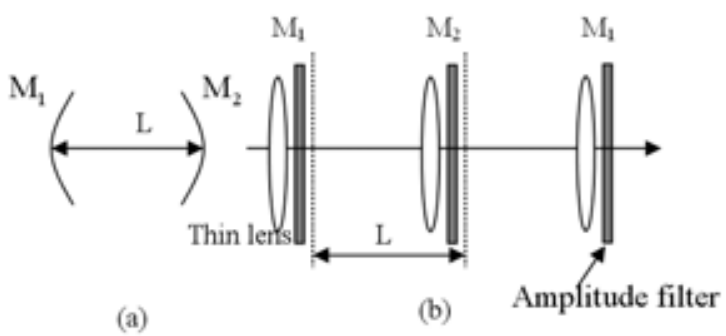

Fig. (9). Schematic diagram of a Gaussian cavity and its equivalent (unfolded) version [77].

By applying the ABCD-matrix approach for a Gaussian aperture [27] we find that if the electromagnetic GSM beam 

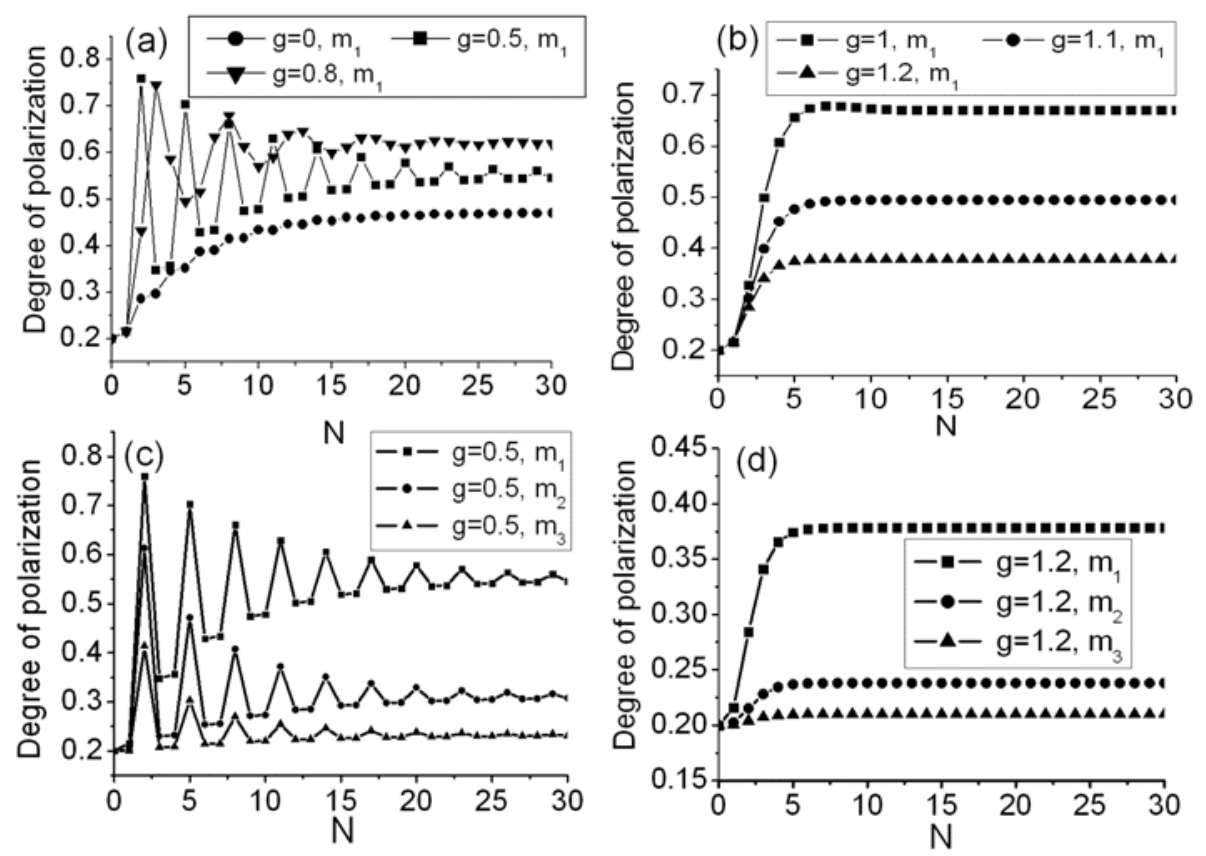

Fig. (10). Degree of polarization (on-axis) versus $N$ for different values of cavity parameter $\mathrm{g}$ and the source correlation coefficients. $m_{1}: \delta_{x}=\delta_{y}=0.1 \mathrm{~mm}, \delta_{x y}=\delta_{y x}=0.2 \mathrm{~mm} . m_{2}: \delta_{x}=\delta_{y}=0.25 \mathrm{~mm}, \delta_{x y}=\delta_{y x}=0.5 \mathrm{~mm} . m_{3}: \delta_{x}=\delta_{y}=0.5 \mathrm{~mm}, \delta_{x y}=\delta_{y x}=1 \mathrm{~mm}$ [77].

travels once between two mirrors [see Fig. (1b)], the matrices $\mathbf{A}, \mathbf{B}, \mathbf{C}$ and $\mathbf{D}$ are

$\left(\begin{array}{ll}\mathbf{A}_{1} & \mathbf{B}_{1} \\ \mathbf{C}_{1} & \mathbf{D}_{1}\end{array}\right)=\left(\left(-\frac{2}{R}-i \frac{\mathbf{I}}{\pi \varepsilon^{2}}\right) \mathbf{I}\left(1-\frac{2 L}{R}-i \frac{\lambda L}{\pi \varepsilon^{2}}\right) \mathbf{I}\right)$

Here the subscript 1 denotes single pass propagation and $\mathcal{E}$ can be regarded as the mirror spot size. After the Nth trip, $\mathbf{A}, \mathbf{B}, \mathbf{C}$ and $\mathbf{D}$ for the equivalent optical system become

$\left(\begin{array}{ll}\mathbf{A} & \mathbf{B} \\ \mathbf{C} & \mathbf{D}\end{array}\right)=\left(\begin{array}{ll}\mathbf{A}_{1} & \mathbf{B}_{1} \\ \mathbf{C}_{1} & \mathbf{D}_{1}\end{array}\right)^{N}$

On substituting from Eq. (52) into Eqs. (8), (49) and (50), we can determine how various statistical properties of an electromagnetic GSM beam evolve in a Gaussian cavity with increased number of passages between the mirrors.

We now study numerically the behavior of the degree of polarization of a typical electroamgnetic GSM beam in a Gaussian cavity. The resonator is characterized by the classical cavity parameter $g=1-L / R$, the resonators with $0 \leq g<1$ being stable and the ones with $g \geq 1$ being unstable. The degree of polarization of the beam is defined by the expression [53-58].

$$
P(\mathbf{r})=\sqrt{1-\frac{4 \operatorname{det} \overleftrightarrow{\mathrm{W}}(\mathbf{r}, \mathbf{r})}{[\operatorname{Tr} \overrightarrow{\mathrm{W}}(\mathbf{r}, \mathbf{r})]^{2}},}
$$

where $\operatorname{Tr}$ denotes the trace of the matrix. In the following numerical examples, the initial beam parameters are chosen to have the following values $\lambda=590 \mathrm{~nm}, A_{x}=A_{y}=0.707$,
$B_{x y}=B_{y x}=0.2$ and $\sigma_{x}=\sigma_{y}=1 \mathrm{~mm}$. In this case the polarization properties are uniform across the source plane with $P(\mathbf{r})=0.2$.

We calculate in Fig. (10) the degree of polarization (onaxis) versus $N$ for different values of cavity parameter $g$ and initial correlation coefficients with $\varepsilon=0.8 \mathrm{~mm}$ One finds from Fig. (10a and b) that the degree of polarization increases as $N$ increase, and its value approaches different constant values for different resonators when $N$ is enough large $(N>30)$. The growth shows an oscillatory behavior in stable resonators $(0 \leq g<1)$. For unstable resonators, the degree of polarization decreases for higher values of $g$. This behavior is similar to that of a spectral shift of a scalar GSM beam in a Gaussian cavity [100]. One finds from Fig. (10c and d) that the degree of polarization decreases as the correlation coefficients in the input plane take larger values both in stable and unstable resonators.

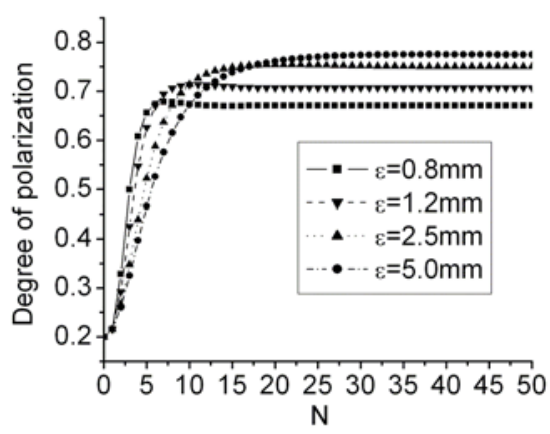

Fig. (11). Degree of polarization (on-axis) versus $N$ for different values of mirror spot size $\varepsilon$ in a Gaussian plane-parallel cavity $(g=1)$ with $\delta_{x}=\delta_{y}=0.1 \mathrm{~mm}$ and $\delta_{x y}=\delta_{y x}=0.2 \mathrm{~mm}$ [77]. 


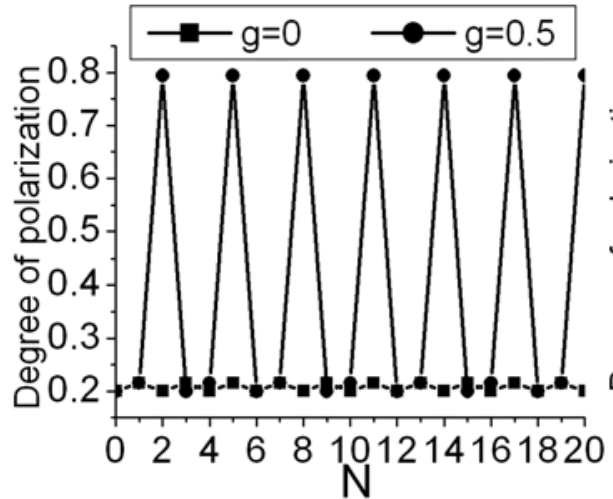

Fig. (12). Degree of polarization (on-axis) versus $N$ for $\delta_{x}=\delta_{y}=0.1 \mathrm{~mm}$ and $\delta_{x y}=\delta_{y x}=0.2 \mathrm{~mm}$

In order to better understand how the mirror spot size $\varepsilon$ affects the degree of polarization, we calculate in Fig. (11) the degree of polarization (on-axis) versus $N$ for different values of $\varepsilon$ in a Gaussian plane-parallel cavity $(g=1)$. We can observe that the growth of the degree of polarization is more pronounced in response to higher values of $\varepsilon$ (i.e., the losses per trip are smaller) when $N$ is sufficiently large.

In the ideal case of a lossless cavity $(\varepsilon \rightarrow \infty)$, we calculate in Fig. (12) the degree of polarization (on-axis) versus $N$ for different values of parameter $g$. One sees that in stable lossless cavities, the degree of polarization has an oscillatory behavior, and its value does not saturate even for large values of $N$. In unstable cavities, the degree of polarization grows rapidly and reaches a saturation value in a few trips, and this value decreases as $g$ increases.

Above discussions were confined to the on-axis degree of polarization. In Fig. (13), we calculate the degree of polarization versus one transverse dimension $x$ for different values of the mirror spot size $\varepsilon$ and the source correlation coefficients in a Gaussian plane-parallel cavity $(g=1)$ with $N=200$. One finds from Fig. (13) that after $\mathrm{N}$ trips in the cavity, an initially uniformly polarized electroamgnetic GSM

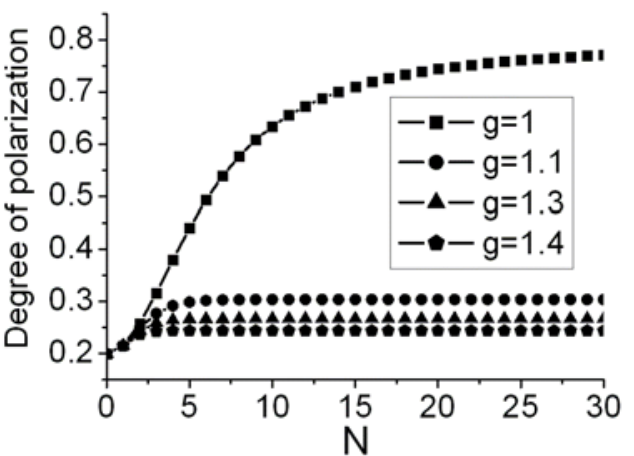

different values of $g$ in a lossless cavity $(\varepsilon \rightarrow \infty)$ with beam has different values of the degree of polarization across the transverse plane of the output mirror and the distribution is Gaussian. We also find that the width of Gaussian distribution is much larger for lower value of $\varepsilon$ (i.e. when the losses per trip are higher) and lower values of the source correlation coefficients. Similar results can be observed also for the cavities with different values of $g$.

From above discussion, we finds that we can control the polarization properties of an electromagnetic GSM beam within a Gaussian cavity by controlling the cavity parameters and the correlation properties of the source. We have also applied our formula to analyze the correlation properties properties and of the state of coherence of an electromagnetic GSM beam in a Gaussian cavity $[77,78]$.

\section{PROPAGATION OF AN ELECTROMAGNETIC GSM BEAM THROUGH PARAXIAL OPTICAL SYSTEM IN TURBULENT ATMOSPHERE}

Within the framework of paraxial approximation, the propagation of the cross-spectral density of a partially coherent beam propagating through a general astigmatic optical system is given by the expression $[101,102]$.

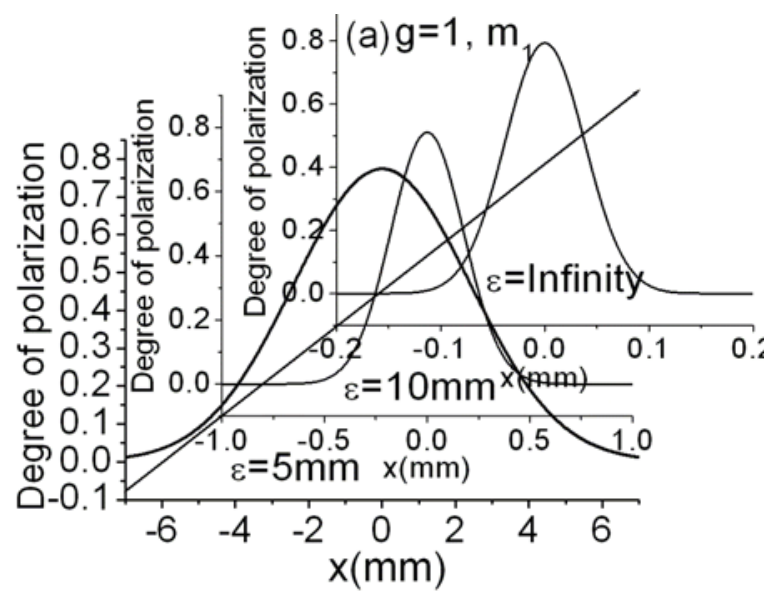

\section{(b) $\mathrm{g}=1, \varepsilon=10 \mathrm{~mm}$}

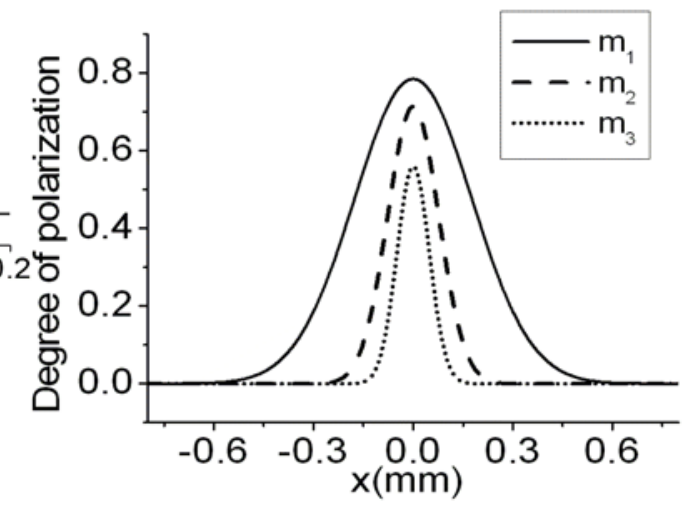

Fig. (13). Degree of polarization versus one transversal dimension $x$ for different values of the mirror spot size $\mathcal{E}$ and the source correlation coefficients in a Gaussian plane-parallel cavity $(g=1) . m_{1}, m_{2}$ and $m_{3}$ takes the same values as used in Fig. (10). 


$$
\begin{aligned}
W\left(\tilde{\mathrm{n}}_{1}, \tilde{\mathrm{n}}_{2}, l\right) & =\frac{1}{\lambda^{2}[\operatorname{det}(\mathbf{B})]^{1 / 2}\left[\operatorname{det}\left(\mathbf{B}^{*}\right)\right]^{1 / 2}} \\
& \int_{-\infty}^{\infty} \int_{-\infty}^{\infty} \int_{-\infty}^{\infty} \int_{-\infty}^{\infty} W\left(\mathbf{r}_{1}, \mathbf{r}_{2}, 0\right)\left\langle\exp \left[\Psi\left(\mathbf{r}_{1}, \tilde{\mathrm{n}}_{1}\right)+\Psi^{*}\left(\mathbf{r}_{2}, \tilde{\mathrm{n}}_{2}\right)\right]\right\rangle \\
& \times \exp \left[-\frac{i k}{2}\left(\mathbf{r}_{1}^{T} \mathbf{B}^{-1} \mathbf{A} \mathbf{r}_{1}-2 \mathbf{r}_{1}^{T} \mathbf{B}^{-1} \tilde{\mathrm{n}}_{1}+\tilde{\mathrm{n}}_{1}^{T} \mathbf{D} \mathbf{B}^{-1} \tilde{\mathrm{n}}_{1}\right)\right] \\
& \times \exp \left[\frac{i k}{2}\left(\mathbf{r}_{2}^{T}\left(\mathbf{B}^{*}\right)^{-1} \mathbf{A}^{*} \mathbf{r}_{2}-2 \mathbf{r}_{2}^{T}\left(\mathbf{B}^{*}\right)^{-1} \tilde{\mathrm{n}}_{2}\right)\right] \\
& \times \exp \left[\frac{i k}{2}\left(\tilde{\mathbf{n}}_{2}^{T} \mathbf{D}^{*}\left(\mathbf{B}^{*}\right)^{-1} \tilde{\mathrm{n}}_{2}\right)\right] d \mathbf{r}_{1} d \mathbf{r}_{2},
\end{aligned}
$$

The term $\left\langle\exp \left[\Psi\left(\mathbf{r}_{1}, \tilde{n}_{1}\right)+\Psi^{*}\left(\mathbf{r}_{2}, \tilde{n}_{2}\right)\right]\right\rangle$ in Eq. (54) is given by Eq. (41). While in this case, the coherence length $\rho_{0}$ is given by the expression $[101,102]$

$\rho_{0}=\operatorname{det}[\mathbf{B}]^{1 / 2}\left(1.46 k^{2} C_{n}^{2} \int_{0}^{l} \operatorname{det}[\mathbf{B}(z)]^{5 / 6} d z\right)^{-3 / 5}$,

Here $\mathbf{B}(z)$ is the sub-matrix for back-propagation from output plane to propagation distance $\mathrm{z}$.

After some arrangement Eq. (4) can be expressed in the tensor form as

$$
\begin{aligned}
W(\tilde{\mathrm{n}}, l) & =\frac{k^{2}}{4 \pi^{2}[\operatorname{det}(\tilde{\mathbf{n}})]^{1 / 2}} \int_{-\infty}^{\infty} \int_{-\infty}^{\infty} \int_{-\infty}^{\infty} \int_{-\infty}^{\infty} W(\tilde{\mathbf{r}}, 0) \\
& \times \exp \left[-\frac{i k}{2}\left(\tilde{\mathbf{r}}^{T} \tilde{\mathbf{B}}^{-1} \tilde{\mathbf{A}} \tilde{\mathbf{r}}-2 \tilde{\mathbf{r}}^{T} \tilde{\mathbf{B}}^{-1} \tilde{\mathbf{n}}+\tilde{\mathbf{n}}^{T} \tilde{\mathbf{D}} \tilde{\mathbf{B}}^{-1} \tilde{\mathbf{n}}\right)\right] \\
& \times \exp \left[-\frac{i k}{2} \tilde{\mathbf{r}}^{T} \tilde{\mathbf{P}} \tilde{\mathbf{r}}-\frac{i k}{2} \tilde{\mathbf{r}}^{T} \tilde{\mathbf{P}} \tilde{\mathbf{n}}-\frac{i k}{2} \tilde{\mathbf{n}}^{T} \tilde{\mathbf{P}} \tilde{\mathbf{n}}\right] d \tilde{\mathbf{r}}
\end{aligned}
$$

where $d \tilde{\mathbf{r}}=d \mathbf{r}_{1} d \mathbf{r}_{2}, \tilde{\mathbf{r}}^{T}=\left(\begin{array}{cc}\mathbf{r}_{1}^{T} & \mathbf{r}_{2}^{T}\end{array}\right), \tilde{\mathbf{n}}^{T}=\left(\begin{array}{cc}\tilde{\mathbf{n}}_{1}^{T} & \tilde{\mathbf{n}}_{2}^{T}\end{array}\right)$ and

$$
\begin{aligned}
\tilde{\mathbf{A}} & =\left(\begin{array}{cc}
\mathbf{A} & 0 \mathbf{I} \\
0 \mathbf{I} & \mathbf{A}^{*}
\end{array}\right), \tilde{\mathbf{B}}=\left(\begin{array}{cc}
\mathbf{B} & 0 \mathbf{I} \\
0 \mathbf{I} & -\mathbf{B}^{*}
\end{array}\right), \tilde{\mathbf{C}}=\left(\begin{array}{cc}
\mathbf{C} & \mathbf{0 I} \\
\mathbf{0 I} & -\mathbf{C}^{*}
\end{array}\right), \\
\tilde{\mathbf{D}} & =\left(\begin{array}{cc}
\mathbf{D} & 0 \mathbf{I} \\
0 \mathbf{I} & \mathbf{D}^{*}
\end{array}\right), \tilde{\mathbf{P}}=\frac{2}{i k \rho_{0}^{2}}\left(\begin{array}{cc}
\mathbf{I} & -\mathbf{I} \\
-\mathbf{I} & \mathbf{I}
\end{array}\right),
\end{aligned}
$$

Substituting from Eq. (47) into Eq. (56), after some vector integration and tensor operations, we obtain the following expression for the elements of the cross-spectral density matrix of an electroamgnetic GSM beam after propagating through an astigmatic ABCD optical system in a turbulent atmosphere

$$
\begin{aligned}
& W_{\alpha \beta}(\tilde{\mathbf{n}}, l)= \frac{A_{\alpha} A_{\beta} B_{\alpha \beta}}{\left[\operatorname{det}\left(\tilde{\mathbf{A}}+\tilde{\mathbf{B}} \mathbf{M}_{0 \alpha \beta}^{-1}+\tilde{\mathbf{B}} \tilde{\mathbf{P}}\right)\right]^{1 / 2}} \\
& \exp \left[-\frac{i k}{2} \tilde{\mathbf{n}}^{T} \mathbf{M}_{1 \alpha \beta}^{-1} \tilde{\mathbf{n}}-\frac{i k}{2} \tilde{\mathbf{n}}^{T} \tilde{\mathbf{P}} \tilde{\mathbf{n}}\right] \\
& \quad \exp \left[-\frac{i k}{2} \tilde{\mathbf{n}}^{T}\left(\tilde{\mathbf{B}}^{-1 T}-\frac{1}{4} \tilde{\mathbf{P}}^{T}\right)\left(\mathbf{M}_{0 \alpha \beta}^{-1}+\tilde{\mathbf{B}}^{-1} \tilde{\mathbf{A}}+\tilde{\mathbf{P}}\right)^{-1} \tilde{\mathbf{P}} \tilde{\mathbf{n}}\right],
\end{aligned}
$$

where

$$
\mathbf{M}_{1 \alpha \beta}^{-1}=\left(\tilde{\mathbf{C}}+\tilde{\mathbf{D}} \mathbf{M}_{0 \alpha \beta}^{-1}+\tilde{\mathbf{D}} \tilde{\mathbf{P}}\right)\left(\tilde{\mathbf{A}}+\tilde{\mathbf{B}} \mathbf{M}_{0 \alpha \beta}^{-1}+\tilde{\mathbf{B}} \tilde{\mathbf{P}}\right)^{-1},
$$

In the absence of turbulence (when $C_{n}^{2}=0$, and hence, $\left.\rho_{0} \rightarrow \infty\right) \tilde{\boldsymbol{P}}=0$. Eq. (58) then reduces to the propagation formula for an electromagnetic GSM beam passing through a general astigmatic $\mathrm{ABCD}$ optical system in free space. If we replace $\mathbf{M}_{0 \alpha \beta}^{-1}$ in Eqs. (58) and (59) with those of anisotropic electromagnetic GSM beam [103] or twised electromagnetic GSM beam [80], then Eqs. (58) and (59) can also be used to study the propagation of such beams through an astigmatic $\mathrm{ABCD}$ optical system in turbulent atmopshere. We have also applied Eqs. (58) and (59) to apply electromagnetic GSM beam for lidar system operating through turbulent atmosphere [79].

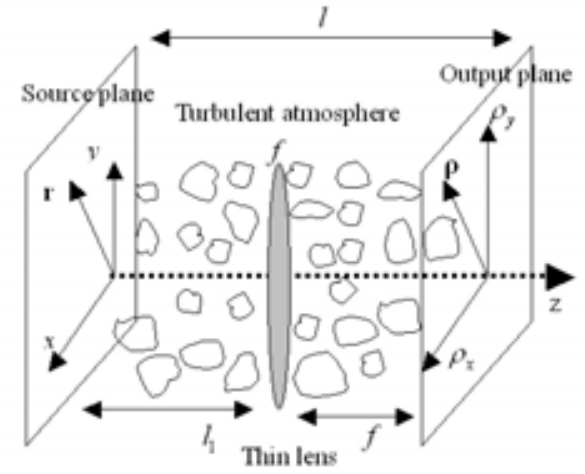

Fig. (14). Focusing geometry [74].

As an application example, now we study the focusing properties of an electromagntic in a turbulent atmosphere. The focusing geometry is shown in Fig. (14). Here the transformation matrix of the total optical system between the source plane and the output plane has the form

$$
\begin{aligned}
\left(\begin{array}{ll}
\mathbf{A} & \mathbf{B} \\
\mathbf{C} & \mathbf{D}
\end{array}\right) & =\left(\begin{array}{cc}
\mathbf{I} & f \mathbf{I} \\
0 & \mathbf{I}
\end{array}\right)\left(\begin{array}{cc}
\mathbf{I} & 0 \mathbf{I} \\
-(1 / f) \mathbf{I} & \mathbf{I}
\end{array}\right)\left(\begin{array}{cc}
\mathbf{I} & l_{\mathbf{I}} \mathbf{I} \\
0 \mathbf{I} & \mathbf{I}
\end{array}\right) \\
& =\left(\begin{array}{cc}
0 \mathbf{I} & f \mathbf{I} \\
-(1 / f) \mathbf{I} & \left(1-l_{1} / f\right) \mathbf{I}
\end{array}\right)
\end{aligned}
$$

For $0<z \leq l_{1}$, the transformation matrix for back-propagation from output plane to plane located at distance $\mathrm{z}$ from the source is given by

$$
\begin{aligned}
\left(\begin{array}{ll}
\mathbf{A}(z) & \mathbf{B}(z) \\
\mathbf{C}(z) & \mathbf{D}(z)
\end{array}\right) & =\left(\begin{array}{cc}
\mathbf{I} & \left(l_{1}-z\right) \mathbf{I} \\
0 & \mathbf{I}
\end{array}\right) \\
& \left(\begin{array}{cc}
\mathbf{I} & 0 \mathbf{I} \\
-(1 / f) \mathbf{I} & \mathbf{I}
\end{array}\right)\left(\begin{array}{cc}
\mathbf{I} & f \mathbf{I} \\
0 \mathbf{I} & \mathbf{I}
\end{array}\right) \\
& =\left(\begin{array}{cc}
\left(1+\frac{z-l_{1}}{f}\right) \mathbf{I} & f \mathbf{I} \\
-(1 / f) \mathbf{I} & 0 \mathbf{I}
\end{array}\right)
\end{aligned}
$$


For $l_{1}<z \leq l_{1}+f$, the transformation matrix for backpropagation from output plane to plane located at distance $\mathrm{z}$ from the source is given by

$$
\left(\begin{array}{ll}
\mathbf{A}(z) & \mathbf{B}(z) \\
\mathbf{C}(z) & \mathbf{D}(z)
\end{array}\right)=\left(\begin{array}{cc}
\mathbf{I} & \left(f+l_{1}-z\right) \mathbf{I} \\
0 \mathbf{I} & \mathbf{I}
\end{array}\right)
$$

Substituting the expression for $\mathbf{B}(z)$ into Eq. (55), we obtain (after integration)

$\rho_{0}=\left[0.1825 C_{n}^{2} k^{2}\left(3 f+8 l_{1}\right)\right]^{-3 / 5}$

The spectral density and the degree of polarization of an electromagnetic GSM beam at point are defined by the expressions

$I\left(\tilde{\mathrm{n}}_{1}, l\right)=\operatorname{Tr} \overrightarrow{\mathrm{W}}\left(\tilde{\mathrm{n}}_{1}, \tilde{\mathrm{n}}_{1}, l\right)$

Now we study the statistical properties of an electromagnetic GSM beams at the geometrical focal plane in a turbulent atmosphere numerically. For all the figures in following text, the parameters of the source of the beam and of the optical system are chosen to be $A_{x}=A_{y}=0.707, B_{x y}=B_{y x}=0.2, f=50 \mathrm{~m}, \lambda=590 \mathrm{~nm}$, $\sigma_{x}=\sigma_{y}=1 \mathrm{~mm}$ and $l_{1}=4.95 \mathrm{~km}$. The polarization properties are uniform across the source plane with $\mathrm{P}\left(\mathbf{r}_{1}, 0\right)=0.2$. We calculate in Figs. (15 and 16) the normalized intensity distribution and corresponding cross line $(y=0)$ of an electroamgnetic GSM beam at the geometrical focal plane for different values of the structure constant of turbulent atmosphere and of the source correlation coefficients. One can see that all the statistical properties of the electromagnetic GSM beam in turbulent atmospheric are closely related to the structure constant $C_{n}^{2}$ and the source correlation coefficients. It is clear from Fig. (15) that the intensity distribution of the electroamgnetic GSM beam at the geometrical focal plane is of Gaussian distribution, and its width increases as the value of the structure constant $C_{n}^{2}$ increases (i.e., the local strength of atmospheric turbulence increases), which shows that an electromagnetic GSM beam can be focused more tightly in free space than in turbulent atmosphere. From Fig. (15d) and Figs. (16a and b) one finds
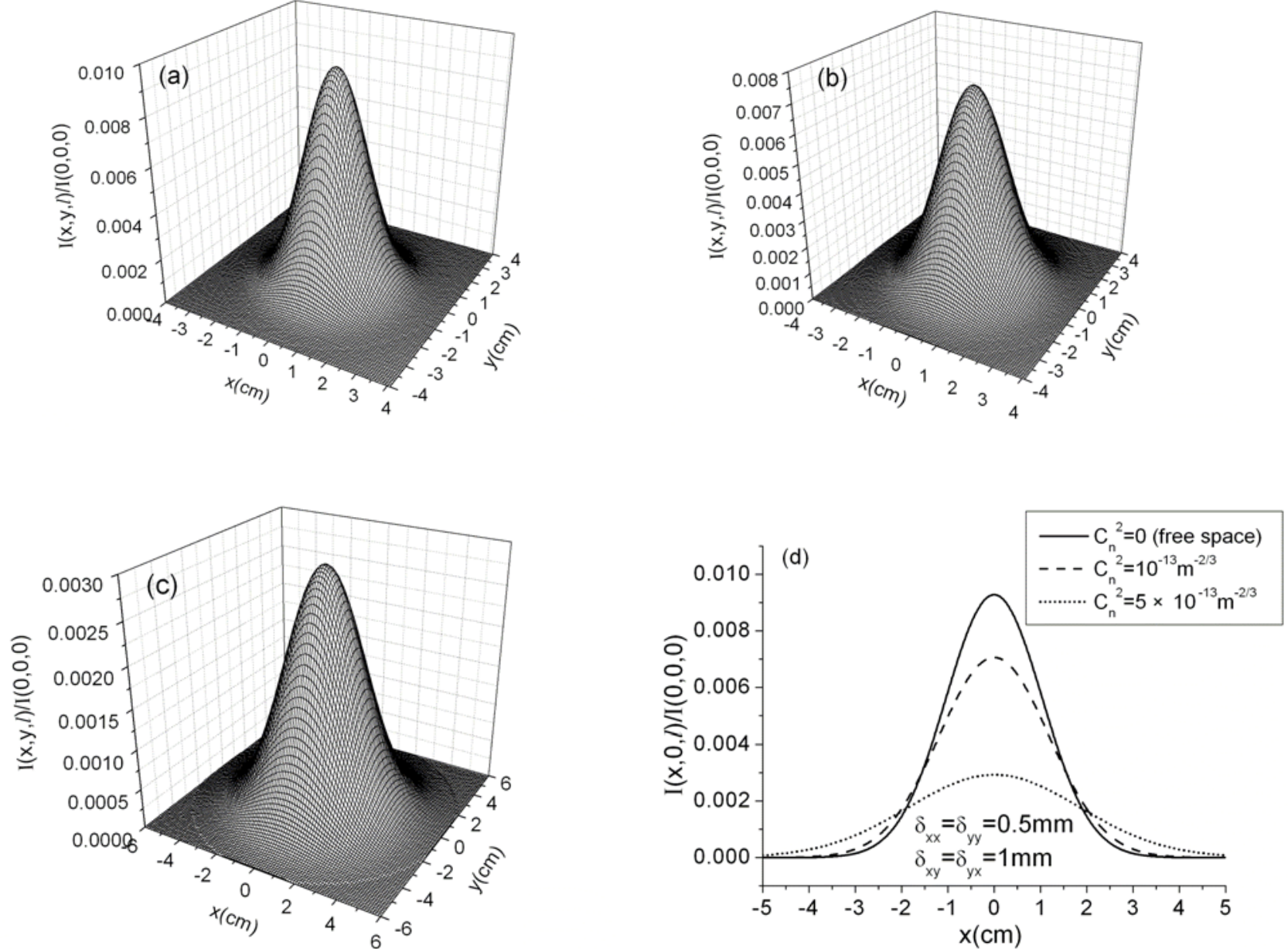

Fig. (15). Normalized intensity distribution and corresponding cross line $(y=0)$ of an electromagnetic GSM beam at the geometrical focal plane for three different values of the structure constant of turbulent atmosphere [74]. 

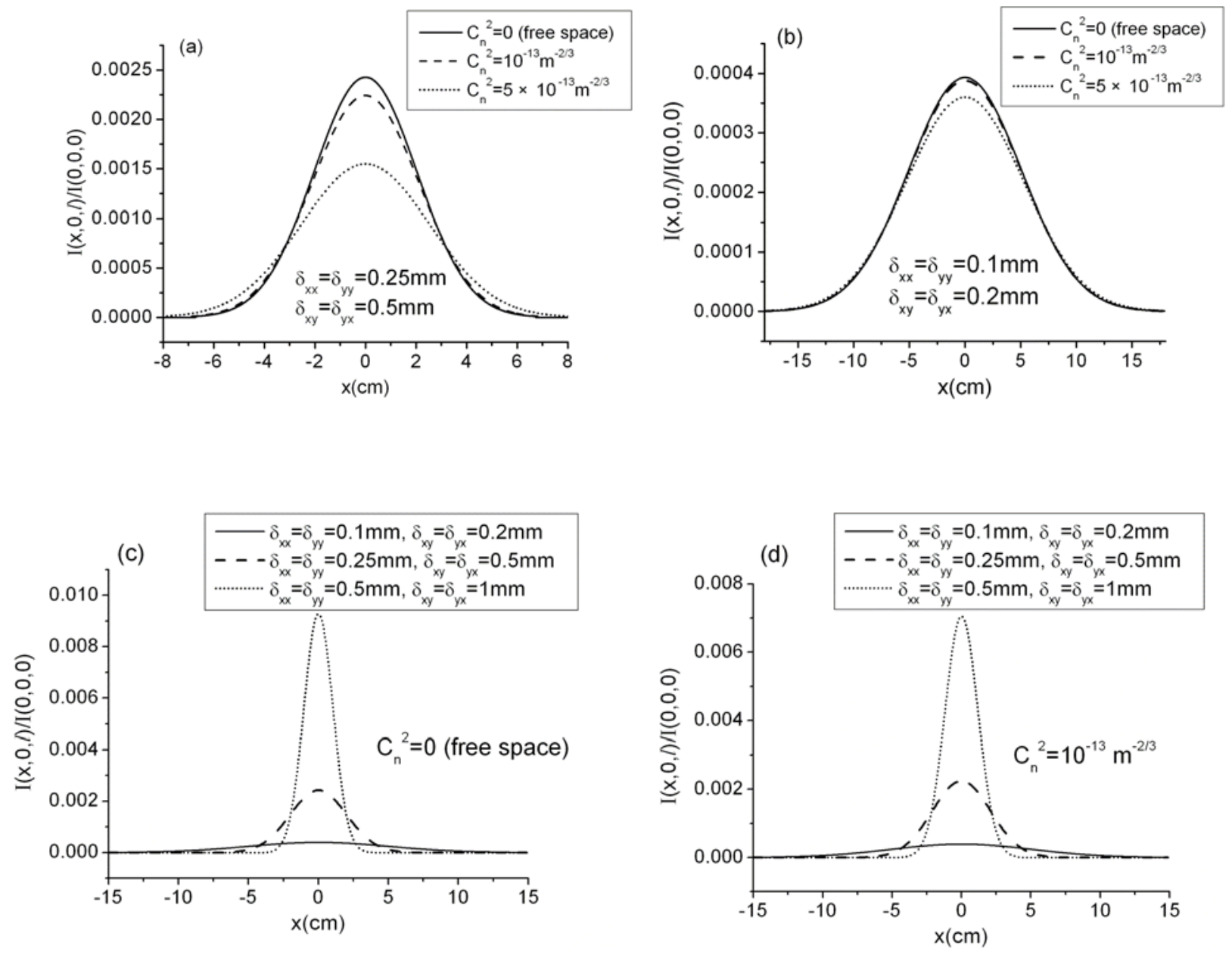

Fig. (16). Normalized intensity distribution (cross line, $y=0$ ) of an electromagnetic GSM beam at the geometrical focal plane for different values of the structure constant of turbulent atmosphere and the source correlation coefficients [74].

that an electromagnetic GSM beam with lower values of the source correlation coefficients is less affected by the atmospheric turbulence than that with higher values of the source correlation coefficients, which is similar to the fact that a scalar GSM beam with lower degree of coherence is less affected by the atmospheric turbulence [2]. One also finds from Figs. (16c and d) that source corre-lation coefficients control the intensity distribution of the focused electromagnetic GSM beam both in free space and in turbulent atmosphere, and an electroamgnetic GSM beam with higher values of the source correlation coefficients can be focused more tightly, which is also similar to the fact that a scalar GSM beam with higher coherence can be focused more tightly [1].

We calculate in Figs. (17 and 18) the degree of polarization and corresponding cross line $(\mathrm{y}=0)$ of an electromagnetic GSM beam at the geometrical focal plane for different values of the structure constant of turbulent atmosphere and the source correlation coefficients. One finds that the initial uniformly polarized electromagnetic GSM beam becomes non-uniformly polarized after focusing, and the degree of polarization is of Gaussian profile. It is evident from Fig. (17) that as the strength of atmospheric turbulence increases, the width of the Gaussian profile increases, the value of the on-axis polarization decreases while the value of the off-axis polarization increases gradually. From Figs. (18a-d), one finds that the shape of the Gaussian profile is affected differently by the refractive index structure parameter $C_{n}^{2}$ and by the source correlation coefficients: with increase in $C_{n}^{2}$ the distribution becomes shorter and flatter, with increase in source correlations it becomes higher and narrower.

\section{CONCLUSION}

In summary, a review of the tensor method for treating the propagation of scalar and electromagnetic Gaussian Schell-model beams was address. We have introuded a tensor method to describe a twisted anisotropic GSM beam and treat its propagation through unapertured and upertured ABCD optical systems, fractional Fourier transform optical system, dispersive and absorbing media and turbulent media. 

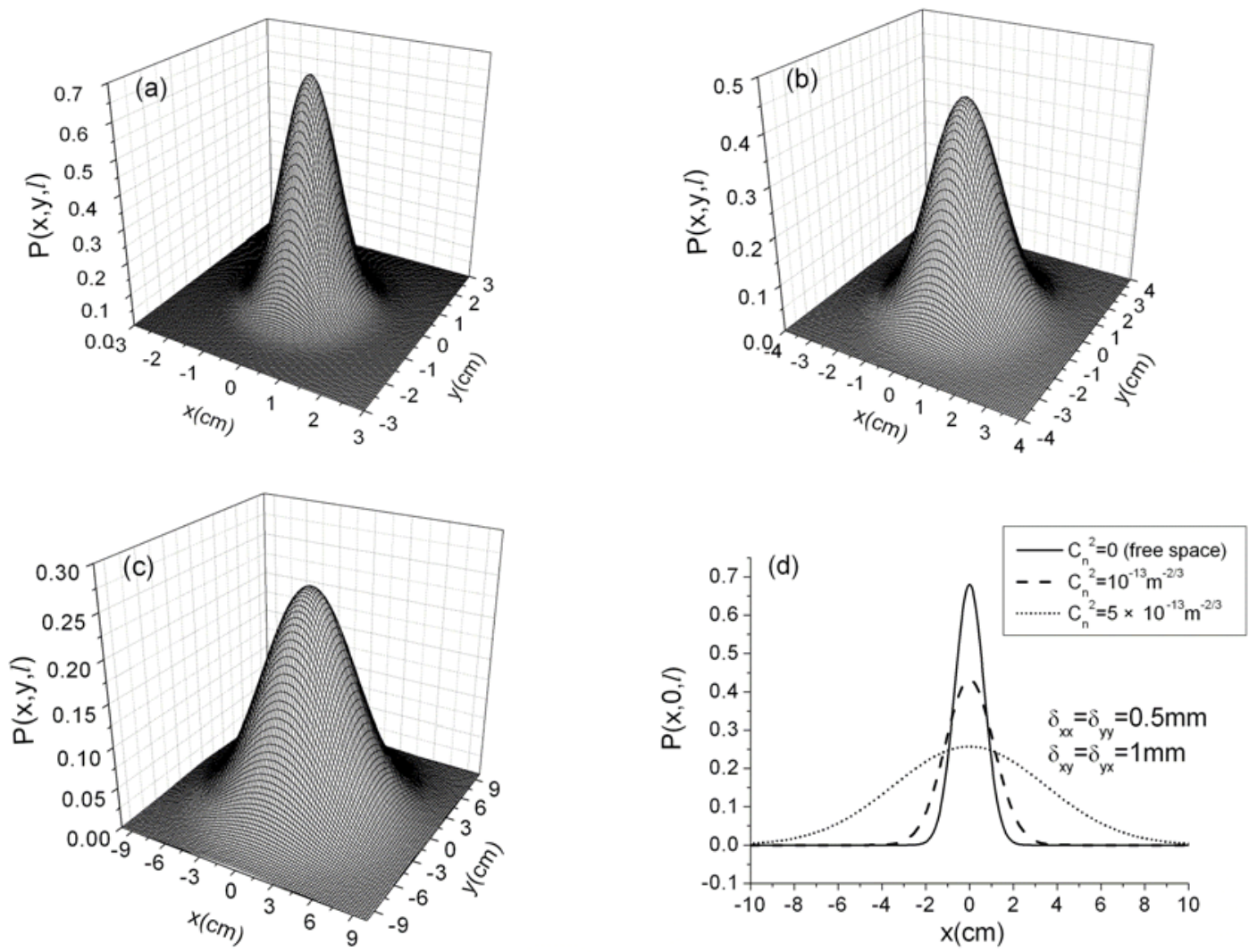

Fig. (17). Degree of polarization and corresponding cross line $(y=0)$ of an electromagnetic GSM beam at the geometrical focal plane for three different values of the structure constant of turbulent atmosphere [74].
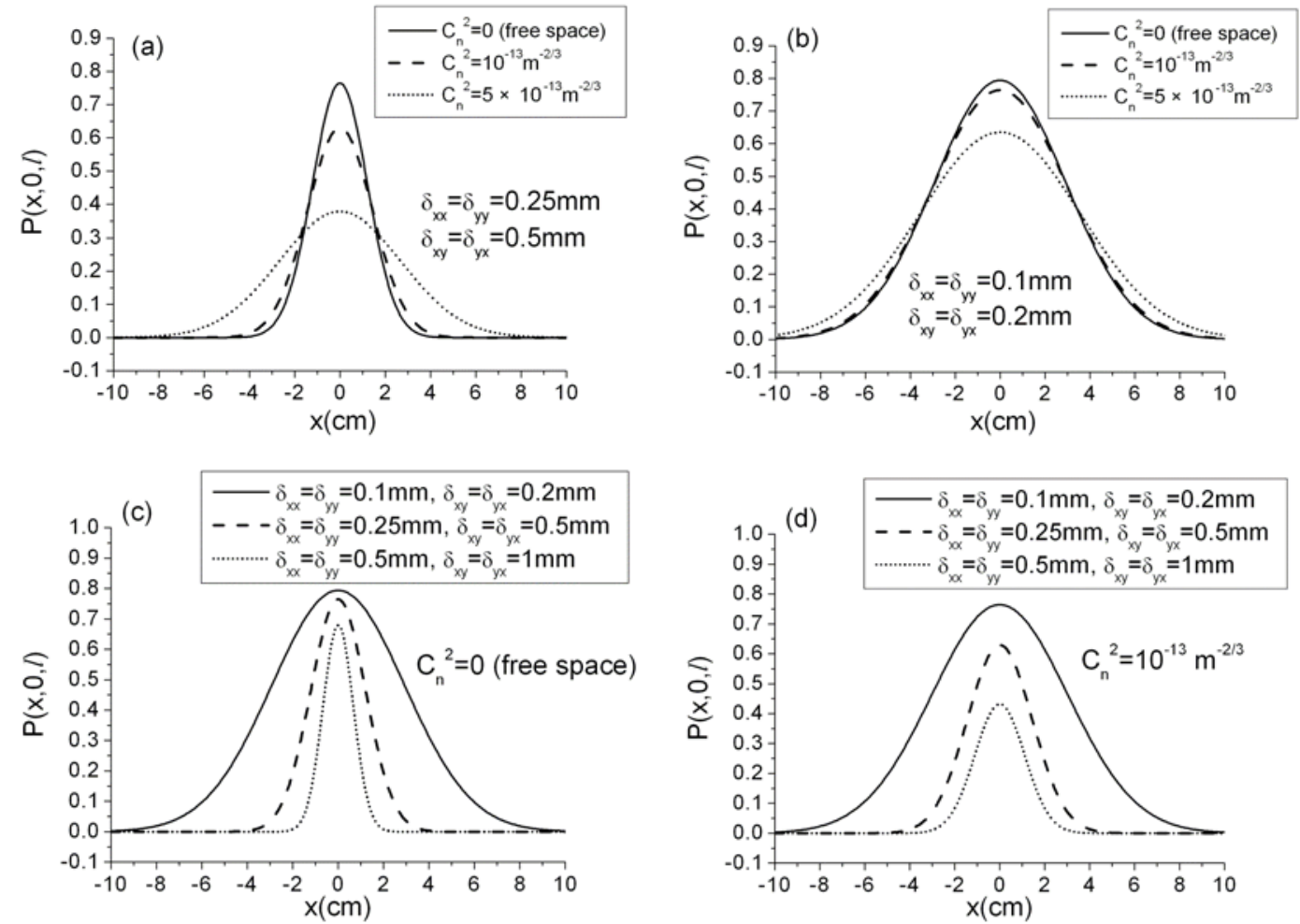

Fig. (18). Degree of polarization (cross line, $y=0$ ) of an electromagnetic GSM beam at the geometrical focal plane for different values of the structure constant of turbulent atmosphere and the source correlation coefficients [74].

We have generated GSM beam experimentally and observed its propagation properties, our experimental results agree well with the theoretical results, which verify the validity of the tensor method. We have also applied the tensor method 
to derive the propagation formulae for an electromagnetic GSM beam passing through a paraxial astigmatic optical system in free space and in turbulent atmosphere, and studied the propagation properties of an electromagnetic GSM beam in a Gaussian cavity and in turbulent atmosphere. The tensor method was used widely to treat the propagation of partially coherent beam with special profiles, such as dark hollow beam, flat-topped beam and laser array beam, and was also used to study the second harmonic generation, ghost imaging and optical trapping with scalar partially coherent beams. We expect to applied the tensor method to study the second harmonic generation, ghost imaging, optical trapping and other applications with electromagnetic partially coherent beams in the future.

\section{REFERENCES}

[1] Mandel L, Wolf E. Optical coherence and quantum optics. New York: Cambridge 1995.

[2] Ricklin JC, Davidson FM. Atmospheric turbulence effects on a partially coherent Gaussian beam: implication for free-space laser communication. J Opt Soc Am A 2002; 19 (9): 1794-802.

[3] Kato Y, Mima K, Miyanaga N, et al. Random phasing of highpower lasers for uniform target acceleration and plasma-instability suppression. Phys Rev Lett 1984; 53(11): 1057-60.

[4] Paganin D, Nugent KA. Noninterferometric phase imaging with partially coherent light. Phys Rev Lett 1998; 80(12): 2586-9.

[5] Gureyev TE, Paganin DM, Stevenson AW, Mayo SC, Wilkins SW. Generalized eikonal of partially coherent beams and its use in quantitative imaging. Phys Rev Lett 2004; 93(6): 068103.

[6] Cai YJ, Zhu SY. Ghost imaging with incoherent and partially coherent light radiation. Phys Rev E 2005; 71(5): 056607.

[7] Cai YJ, Zhu SY. Ghost interference with partially coherent radiation, Opt Lett 2004; 29(23): 2716-8.

[8] Belendez A, Carretero L, Fimia A. The use of partially coherent light to reduce the efficiency of silver-halide noise gratings. Opt Commun 1993; 98(4-6): 236-40.

[9] Dente GC, Osgood JS. Some observations of the effects of partial coherence on projection system imagery. Opt Eng 1983; 22 (6): $720-4$.

[10] Zubairy MS, McIver JK. Second-harmonic generation by a partially coherent beam. Phys Rev A 1987; 36(1): 202-6.

[11] Cai YJ, Peschel U, Second-harmonic generation by an astigmatic partially coherent beam. Opt Express 2007; 15 (23): 15480-92.

[12] Cheng CF, Liu WX, Gui WL. Diffraction halo function of partially coherent speckle photography. Appl Opt 1999; 38 (32): 6687-91.

[13] Zhao C, Cai YJ, Lu X, Eyyuboğlu HT. Radiation force of coherent and partially coherent flat-topped beams on a Rayleigh particle. Opt Express 2009; 17(3): 1753-65.

[14] Wolf E. Invariance of the spectrum of light on propagation. Phys Rev Lett 1986; 56(13): 1370-2.

[15] Wolf E. Non-cosmological redshifts. Nature 1987; 326: 363-5.

[16] Wolf E. Red shifts and blue shifts of spectral lines emitted by two correlated sources. Phys Rev Lett 1987; 58(25): 2646-8.

[17] Wolf E, Collett E. Partially coherent sources which produce same far-field intensity distribution as a laser. Opt Commun 1978; 25(3): 293-6.

[18] Santis PD, Gori F, Guattari G, Palma C. An example of a CollettWolf source. Opt Commun 1979; 29(2): 256-60.

[19] Gori F. Collet-Wolf sources and multimode lasers. Opt Commun 1980; 34 (3): 301-5.

[20] Friberg AT, Sudol RJ. Propagation parameters of Gaussian Schellmodel beams. Opt Commun 1982; 41(6): 383-7.

[21] Friberg AT, Turunen J. Imaging of Gaussian Schell-model sources. J Opt Soc Am A 1988; 5(5): 713-20.
[22] Wang F, Cai YJ. Experimental observation of fractional Fourier transform for a partially coherent optical beam with Gaussian statistics. J Opt Soc Am A 2007; 24(7): 1937-44.

[23] Tervonen E, Friberg AT, Turunen J. Gaussian Schell-model beams generated with synthetic acousto-optic holograms. J. Opt Soc Am A 1992; 9(5): 796-803.

[24] Simon R, Mukunda N. Twisted Gaussian Schell-model beams. J Opt Soc Am A 1993; 10(1): 95-109.

[25] Simon R, Sundar K, Mukunda N. Twisted Gaussian Schell-model beams. I. Symmetry structure and normal-mode spectrum. J Opt Soc Am A 1993; 10(9): 2008-16.

[26] Sundar K, Simon R, Mukunda N. Twisted Gaussian Schell-model beams. II. Spectrum analysis and propagation characteristics. J Opt Soc Am A 1993; 10(9): 2017-23.

[27] Simon R, Mukunda N. Twist phase in Gaussian-beam optics. J Opt Soc Am A 1998; 15(9): 2373-82

[28] Simon R, Mukunda N. Gaussian Schell-model beams and general shape invariance. J Opt Soc Am A 1999; 16(10): 2465-75.

[29] Ambrosini D, Bagini V, Gori F, Santarsiero M. Twisted Gaussian Schell-model beams: a superposition model. J Mod Opt 1994; 41(7): 1391-9.

[30] Friberg AT, Tervonen E, Turunen J. Interpretation and experimental demonstration of twisted Gaussian Schell-model beams. J Opt Soc Am A 1994; 11(6): 1818-26.

[31] Sundar K, Mukunda N, Simon R. Coherent-mode decomposition of general anisotropic Gaussian Schell-model beams. J Opt Soc Am A 1995; 12(3): 560-9.

[32] Simon R, Friberg AT, Wolf E. Transfer of radiance by twisted Gaussian Schell-model beams in paraxial systems. Pure Appl Opt 1996; 5 (3): 331-43.

[33] Östlund P, Friberg AT. Radiometry and radiation efficiency of twisted Gaussian Schell-model Sources. Opt Rev 2001; 8(1): 1-8.

[34] Serna J, Movilla JM. Orbital angular momentum of partially coherent beams. Opt Lett 2001; 26(7): 405-6.

[35] Bastiaans MJ. Application of the Wigner distribution function to partially coherent light. J Opt Soc Am A 1986; 3(8): 1227-38.

[36] Bastiaans MJ. Wigner-distribution function applied to twisted Gaussian light propagating in first-order optical systems. J Opt Soc Am A 2000; 17(12): 2475-80.

[37] Lin Q, Cai YJ. Tensor ABCD law for partially coherent twisted anisotropic Gaussian-Schell model beams. Opt Lett 2002; 27(4): 216-8.

[38] Cai YJ, Lin Q. Propagation of partially coherent twisted anisotropic Gaussian Schell-model beams through misaligned optical systems. Opt Commun 2002; 211(1-6): 1-8.

[39] Cai YJ, Lin Q, Focusing properties of partially coherent twisted anisotropic Gaussian-Schell model beams. Opt Commun 2003; 215(4-6): 239-45.

[40] Cai YJ, Huang YX, Lin Q. Spectral shift of partially coherent twisted anisotropic Gaussian-Schell-model beams focused by a thin lens. J Opt A: Pure Appl Opt 2003; 5(4): 397-401.

[41] Cai YJ, Lin Q. Spectral shift of partially coherent twisted anisotropic Gaussian Schell-model beams in free space. Opt Commun 2002; 204(1-6): 17-23.

[42] Cai YJ, Hu L. Propagation of partially coherent twisted anisotropic Gaussian Schell-model beams through an apertured astigmatic optical system. Opt Lett 2006; 31(2): 685-7.

[43] Lin Q, Cai YJ. Fractional Fourier transform for partially coherent Gaussian-Schell model beams. Opt Lett 2002; 27(19): 1672-4.

[44] Cai YJ, Lin Q. Transformation and spectrum properties of partially coherent beams in the fractional Fourier transform plane. J Opt Soc Am 2003; 20(8): 1528-36.

[45] Cai YJ, Lin Q, Ge D. Propagation of partially coherent twisted anisotropic Gaussian Schell-model beams in dispersive and absorbing media. J Opt Soc Am A 2002; 19(10): 2036-42.

[46] Cai YJ, He SL. Propagation of a partially coherent twisted anisotropic Gaussian Schell-model beam in a turbulent atmosphere Appl Phys Lett 2006; 89(4): 041117.

[47] Cai YJ, Lin Q, Korotkova O. Ghost imaging with twisted Gaussian Schell-model beam. Opt Express 2009; 17(4): 2450-64. 
[48] Brosseau C. Fundamentals of polarized light-A statistical approach. New York: Wiley 1995.

[49] James DFV. Changes of polarization of light beams on propagation in free space. J Opt Soc Am A 1994; 11(5): 1641-3.

[50] Gori F. Matrix treatment for partially polarized partially coherent beams. Opt Lett 1998; 23(4): 241-3.

[51] Agrawal GP, Wolf E. Propagation-induced polarization changes in partially coherent optical beams. J Opt Soc Am A 2000; 17(11): 2019-23.

[52] Gori F, Santarsiero M, Vicalvi S, Borghi R, Guattari G. Beam coherence-polarization matrix. Pure Appl Opt 1998; 7(5): 941-51.

[53] Gori F, Santarsiero M, Piquero G, Borghi R, Mondello A, Simon R. Partially polarized Gaussian schell-model beams. J Opt A: Pure Appl Opt 2001; 3(1): 1-9.

[54] Wolf E. Unified theory of coherence and polarization of random electromagnetic beams. Phys Lett A 2003; 312(5-6): 263-7.

[55] Wolf E. Introduction to the theory of coherence and polarization of light. Cambridge: Cambridge University Press 2007.

[56] Piquero G, Gori F, Romanini P, Santarsiero M, Borghi R, Mondello A. Synthesis of partially polarized Gaussian Schell-model sources. Opt Commun 2002; 208(1-3): 9-16.

[57] Shirai T, Korotkova O, Wolf E. A method of generating electromagnetic Gaussian Schell-model beams. J Opt A: Pure Appl Opt 2005; 7(5): 232-7.

[58] Korotkova O, Salem M, Wolf E. Beam conditions for radiation generated by an electromagnetic Gaussian Schell-model source. Opt Lett 2004; 29(11): 1173-5.

[59] Gori F, Santarsiero M, Borghi R, Ramírez-Sánchez V. Realizability condition for electromagnetic Schell-model sources. J Opt Soc Am A 2008; 25(5): 1016-21.

[60] Korotkova O, Wolf E. Changes in the state of polarization of a random electromagnetic beam on propagation. Opt Commun 2005; 246(1-3): 35-43.

[61] Korotkova O, Wolf E. Effects of linear non-image-forming devices on spectra and on coherence and polarization properties of stochastic electromagnetic beams: part I: general theory. J Mod Opt 2005; 52(18): 2659-71.

[62] Korotkova O, Salem M, Wolf E. The far-zone behavior of the degree of polarization of electromagnetic beams propagating through atmospheric turbulence. Opt Commun 2004; 233(4-6): 22530.

[63] Roychowdhury H, Ponomarenko SA, Wolf E. Change in the polarization of partially coherent electromagnetic beams propagating through the turbulent atmosphere. J Mod Opt 2005; 52(11): 1611-8.

[64] Eyyuboğlu HT, Baykal Y, Cai Y. Degree of polarization for partially coherent general beams in turbulent atmosphere. Appl Phys B 2007; 89(1): 91-7.

[65] Gao W. Changes of polarization of light beams on propagation through tissue. Opt Commun 2006; 260(2): 749-54.

[66] Gao W, Korotkova O. Changes in the state of polarization of a random electromagnetic beam propagating through tissue. Opt Commun 2007; 270(2): 474-8.

[67] Korotkova O, Wolf E. Generalized stokes parameters of random electromagnetic beams. Opt Lett 2005; 30 (2): 198-200

[68] Korotkova O. Changes in statistics of the instantaneous stokes parameters of a quasi-monochromatic electromagnetic beam on propagation. Opt Commun 2006; 261(2): 218-24.

[69] Korotkova O. Scintillation index of a stochastic electromagnetic beam propagating in random media. Opt Commun 2008; 281(9): 2342-8.

[70] Salem M, Wolf E. Coherence-induced polarization changes in light beams. Opt Lett 2008; 33(11): 1180-2

[71] Roychowdhury H, Agrawal GP, Wolf E. Changes in the spectrum, in the spectral degree of polarization, and in the spectral degree of coherence of a partially coherent beam propagating through a gradient-index fiber. J Opt Soc Am A 2006; 23(4): 940-8.

[72] Cai YJ, Ge D, Lin Q. Fractional fourier transform for partially coherent and partially polarized Gaussian Schell-model beams. J Opt A: Pure Appl Opt 2003; 5(5): 453-9.
[73] Ge D, Cai YJ, Lin Q. Propagation of partially polarized Gaussian Schell-model beams through aligned and misaligned optical system. Chin Phys 2005; 14(1): 128-32.

[74] Cai YJ, Korotkova O, Eyyuboğlu HT, Baykal Y. Active laser radar systems with stochastic electromagnetic beams in turbulent atmosphere. Opt Express 2008; 16(20): 15835-46.

[75] Ge D, Cai YJ, Lin Q. Propagation of partially polarized Gaussian Schell-model beams in dispersive and absorbing media. Opt Commun 2004; 229(1-6): 93-8.

[76] Yao M, Cai YJ, Eyyuboğlu HT, Baykal Y, Korotkova O. The evolution of the degree of polarization of an electromagnetic Gaussian Schell-model beam in a Gaussian cavity. Opt Lett 2008; 33(19): 2266-8

[77] Korotkova O, Yao M, Cai YJ, Eyyuboğlu HT, Baykal Y. The state of polarization of a stochastic electromagnetic beam in an optical resonator. J Opt Soc Am A 2008; 25(11): 2710-20.

[78] Tong Z, Korotkova O, Cai YJ, Eyyuboglu HT, Baykal Y. Correlation properties of random electromagnetic beams in laser resonators. Appl Phys B 2009; doi:10.1007/s00340-009-3629-2.

[79] Korotkova O, Cai YJ, Watson E. Stochastic electromagnetic beams for Lidar systems operating through turbulent atmosphere. Appl Phys B 2009; 94(4): 681-90.

[80] Cai YJ, Korotkova O. Twist phase-induced polarization changes in electromagnetic Gaussian Schell-model beams. Appl Phys B 2009; 96(2-3): 499-507.

[81] Li Y, Wolf E. Focal shift in diffracted coverging spherical waves. Opt Commun 1981; 39(4): 211-5.

[82] Li Y. Focal shift and focal switch in dual focus systems. J Opt Soc Am A 1997; 14(6): 1297-304.

[83] Pu J, Zhang H, Nemoto S. Spectral shifts and spectral switches of partially coherent light passing through an aperture. Opt Commun 1999; 162(1-3): 57-63.

[84] Wen JJ, Breazeale MA. A diffraction beam field expressed as the superposition of Gaussian beams. J Acoust Soc Am 1988; 83(5): $1752-6$.

[85] Mendlovic D, Ozaktas HM. Fractional fourier transforms and their optical implementation: I. J Opt Soc Am A 1993; 10(9): 1875-81.

[86] Lohmann AW. Image rotation, wigner rotation, and the fractional fourier transform. J Opt Soc Am A 1993; 10(10): 2181-6.

[87] Lohmann AW, Medlovic D, Zalevsky Z. Fractional transformations in optics. In: Wolf E, Ed. Progress in Optics. Amsterdam: Elsevier 1998; Vol. 38.

[88] Ozaktas HM, Zalevsky Z, Kutay MA. The fractional fourier transform with applications in optics and signal processing. New York: Wiley 2001.

[89] Wang F, Cai YJ, He SL. Experimental observation of the coincidence fractional Fourier transform with a partially coherent beam. Opt Express 2006; 14 (16): 6999-7004.

[90] Wang F, Cai YJ. Experimental observation of fractional fourier transform for a partially coherent optical beam with Gaussian statistics. J Opt Soc Am A 2007; 24(7): 1937-44.

[91] Wang F, Cai YJ, Lin Q. Experimental observation of truncated fractional fourier transform for a partially coherent Gaussian Schellmodel beam. J Opt Soc Am A 2008; 25(8): 2001-10.

[92] Ishimaru A. Wave propagation and scattering in random media New York: Academic Press 1978.

[93] Andrews LC, Phillips RL. Laser beam propagation through random media. Bellingham: SPIE 1998.

[94] Cai YJ, Lin Q, Eyyuboğlu HT, Baykal Y. Off-axis Gaussian Schellmodel beam and partially coherent laser array beam in a turbulent atmosphere. Opt Commun 2007; 278 (1): 157-67.

[95] Cai YJ, Chen YT, Eyyuboğlu HT, Baykal Y. Propagation of laser array beams in a turbulent atmosphere. Appl Phys B 2007; 88 (3): 467-75.

[96] Cai YJ, He SL. Average intensity and spreading of an elliptical Gaussian beam propagating in a turbulent atmosphere. Opt Lett 2006; 31(5): 568-70.

[97] Cai YJ, He SL. Propagation of various dark hollow beams in a turbulent atmosphere. Opt Express 2006; 14 (4): 1353-67. 
[98] Cai YJ. Propagation of various flat-topped beams in a turbulent atmosphere. J Opt A: Pure Appl Opt 2006; 8 (6): 537-45.

[99] Cai YJ, Chen YT, Eyyuboğlu HT, Baykal Y. Scintillation index of elliptical Gaussian beam in turbulent atmosphere. Opt Lett 2007; 32 (16): 2405-7.

[100] Palma C, Cardone G, Cincotti G. Spectral changes in Gaussiancavity lasers. IEEE J Quant Electron 1998; 34(7): 1082-8.
[101] Yura HT, Hanson SG. Optical beam wave propagation through complex optical systems. J Opt Soc Am A 1987; 4 (10): 1931-48.

[102] Yura HT, Hanson SG. Second-order statistics for wave propagation through complex optical systems. J Opt Soc Am A 1989; 6(4): 56475.

[103] Wang H, Wang X, Zeng A, Yang K. Effects of coherence on anisotropic electromagnetic Gaussian-Schell model beams on propagation. Opt Lett 2007; 32(15): 2215-7.

Received: September 07, 2009

Revised: November 30, 2009

Accepted: December 02, 2009

(c) Cai and Wang; Licensee Bentham Open.

This is an open access article licensed under the terms of the Creative Commons Attribution Non-Commercial License (http://creativecommons.org/licenses/by$\mathrm{nc} / 3.0 /$ ), which permits unrestricted, non-commercial use, distribution and reproduction in any medium, provided the work is properly cited. 